\title{
Embedded electromagnetically sensitive particle motion in functionalized fluids
}

\section{T. Zohdi}

Received: 28 January 2014 / Accepted: 28 March 2014 / Published online: 24 April 2014

(C) Springer International Publishing Switzerland 2014

\begin{abstract}
The primary objective of this paper is to characterize the motion of small electromagnetically sensitive particles which are embedded in a flowing neutral fluid. There are a variety of industrial applications for electromagnetic particle-laden fluids, such as fluid-based actuators, coatings and functionalized inks, to name a few. This work compares the relative strengths of the forces induced by electromagnetic fields and fluid drag, and their composite effects on particle motion. Both analytical and numerical investigations are undertaken. After an analysis of a single isolated particle, a three-dimensional model problem comprised of a Representative Volume Element of flowing particle-laden fluid, under the action of external electromagnetic fields, is studied. A computational staggering scheme is developed to solve the coupled system utilizing a fully implicit Finite-Difference discretization of the Navier-Stokes equations for the fluid and a direct particledynamics description is used for the particles. For large numbers of embedded particles, because of the extreme computational difficulty of interface-conforming fine-mesh spatial discretizations for the fluid, simplifying assumptions for the coupling are made based on semi-analytical computation of drag-coefficients, allowing for the use of coarser meshes. Even after these simplifications, the particle-fluid system is strongly-coupled. The strongly-coupled system is implicitly solved, iteratively, within each time-step using a recursive staggering scheme, which employs temporal adaptivity to control the coupling error. The approach allows researchers to rapidly compute such systems with moderate laptop/desktop resources. Numerical examples are provided to illustrate the
\end{abstract}

T. Zohdi $(\varangle)$

Department of Mechanical Engineering, University of California, 6195 Etcheverry Hall, Berkeley, CA 94720-1740, USA

e-mail: zohdi@berkeley.edu model and the numerical solution scheme, and limitations and extensions of the model are discussed.

Keywords Particle-laden fluids $\cdot$ Reduced-order coupling · Electromagnetism $\cdot$ Particles $\cdot$ Fluids

\section{Applications}

Electromagnetically sensitive fluids are typically constructed ("functionalized") by embedding charged or magnetic particles in a neutral fluid. Such fluids date back, at least, to Winslow [45,46] in 1947. The most widely used class of such fluids are electrorheological fluids, which are comprised of extremely fine suspensions of charged particles (on the order of 50 microns) in an electrically neutral fluid. ${ }^{1}$ They have been historically used in specialized niche applications involving hydraulic valves, actuators, flexible screens, brakes, liquid seals and recently in electromagnetically sensitive inks (so-called e-inks), for large-scale printing applications and coatings. Furthermore, they have been proposed for use in many other evolving applications, such as targeted drug-delivery and large-scale shape shifting mirrors. A particular recent application, which has motivated this analysis (Zohdi [57]), is the controlled localized interior wall growth in specific damaged locations within piping systems, by electromagnetically guiding charged particles that are embedded in the fluid to those locations. The overall objective is to heal damaged large-scale industrial piping systems noninvasively, from the exterior, during operation, resulting in no down time and reduction of repair costs. The first step in achieving this overall goal is the analysis of the movement of seeded charged particles. In

\footnotetext{
${ }^{1}$ Later in the paper we also discuss ferro-fluids.
} 
such a system, there are two primary effects that play a role in the charged particle dynamics: (1) drag forces due to the fluid flow and (2) electromagnetic forces due to an external source. It is the goal of this research to develop and study the relationships that characterize both contributions. $^{2}$

The main objective of this communication is to characterize the motion of electromagnetically sensitive small-scale particles embedded in a flowing neutral fluid. Initially, the dynamics of a single particle is studied. Thereafter, a threedimensional Representative Volume Element of a flowing particle-laden fluid, under the action of external electromagnetic fields, is investigated. A fully implicit Finite-Difference discretization of the Navier-Stokes equations is used for the fluid and a direct particle-dynamics discretization is performed for the particles. Because of the large computational difficulty and expense of a conforming spatial discretization needed for large numbers of embedded particles, simplifying assumptions are made for the coupling, based on semianalytical computation of drag-coefficients, which allows for the use of coarser meshes. Even after these simplifications, the particle-fluid system is strongly-coupled. The approach taken in the present work is to construct a sub-model for each primary physical process. In order to resolve the coupling, a recursive staggering scheme is constructed, which builds on works found in Zohdi [50,51,55,56,58-62]. The procedure is as follows (at a given time increment): (1) each submodel equation (fluid or particle-system) is solved individually, "freezing" the other (coupled) fields in the system, allowing only the primary field to be active, (2) after the solution of each submodel, the associated field variable is updated, and the next submodel is solved and (3) the process is then repeated, until convergence. The time-steps are adjusted to control the rates of convergence, which is dictated by changes in the overall physics. Specifically, the approach is a staggered implicit time-stepping scheme, with an internal recursion that automatically adapts the time-step sizes to control the rates of convergence within a time-step. If the process does not converge (below an error tolerance) within a preset number of iterations, the time-step is adapted (reduced) by utilizing an estimate of the spectral radius of the coupled system. The modular approach is designed for easy replacement of submodels for the fluid, particles and their interaction. The approach allows researchers to rapidly compute such systems with laptop/desktop resources. Numerical examples are provided to illustrate the model and numerical solution scheme, and limitations and extensions of the approach are discussed.

\footnotetext{
$\overline{2}$ More details on this specific application in mind are given in the conclusions.
}

\section{An isolated particle}

\subsection{Particle dynamics in an electromagnetic field}

We recall the following important observations in conjunction with electromagnetic phenomena (Jackson [17]):

- If a point charge $q$ experiences a force $\boldsymbol{\Psi}^{e}$, the electric field, $\boldsymbol{E}^{e x t}$, at the location of the charge is defined by $\boldsymbol{\Psi}^{e}=$ $q \boldsymbol{E}^{\text {ext }}$.

- If the charge is moving, another force may arise, $\Psi^{m}$, which is proportional to its velocity $\boldsymbol{v}$. This other (induced) field is denoted as the "magnetic induction" or just the "magnetic field", $\boldsymbol{B}^{\text {ext }}$, such that $\boldsymbol{\Psi}^{m}=q \boldsymbol{v} \times \boldsymbol{B}^{\text {ext }}$.

- If the forces occur concurrently (the charge is moving through the region possessing both electric and magnetic fields), then the electromagnetic force is $\boldsymbol{\Psi}^{e+m}=$ $q \boldsymbol{E}^{e x t}+q \boldsymbol{v} \times \boldsymbol{B}^{\text {ext }}$.

We consider an isolated charged mass with position vector denoted by $\boldsymbol{r}$, governed by $(\dot{\boldsymbol{r}}=\boldsymbol{v}, \ddot{\boldsymbol{r}}=\dot{\boldsymbol{v}})$

$m \dot{\boldsymbol{v}}=q\left(\boldsymbol{E}^{e x t}+\boldsymbol{v} \times \boldsymbol{B}^{e x t}\right)$.

The governing Eq. 2.1, written in component form is, for component 1

$\dot{v}_{1}=\frac{q}{m}\left(E_{1}^{e x t}+\left(v_{2} B_{3}^{e x t}-v_{3} B_{2}^{e x t}\right)\right)$,

for component 2

$\dot{v}_{2}=\frac{q}{m}\left(E_{2}^{e x t}-\left(v_{1} B_{3}^{e x t}-v_{3} B_{1}^{e x t}\right)\right)$,

and for component 3

$\dot{v}_{3}=\frac{q}{m}\left(E_{3}^{e x t}+\left(v_{1} B_{2}^{e x t}-v_{2} B_{1}^{e x t}\right)\right)$.

If we make the simplifying assumption that $\boldsymbol{E}^{\text {ext }}=\left(E_{1}^{\text {ext }}\right.$, $E_{2}^{e x t}, E_{3}^{e x t}$ ) is an independent (not dependent on the particles) external electric field and $\boldsymbol{B}^{\text {ext }}=\left(B_{1}^{\text {ext }}, B_{2}^{\text {ext }}, B_{3}^{\text {ext }}\right)$ is an independent external magnetic field, the equations can be solved analytically (for example, see Jackson [17]) for an isolated particle (Fig. 1). The electric and magnetic fields are assumed to be independent of one another. We consider a few of these cases, for static ("dead") electromagnetic fields.

\subsubsection{Qualitative electromagnetically driven dynamics of a charged particle without fluid drag}

Following Zohdi [61,62], we consider both the electric and magnetic fields to be present, $\boldsymbol{r}(t=0)=\mathbf{0}, \boldsymbol{v}(t=0)=$ $v_{o} \boldsymbol{e}_{1}, \boldsymbol{B}^{\text {ext }}=B_{3}^{\text {ext }} \boldsymbol{e}_{3}$ and $\boldsymbol{E}^{\text {ext }}=E_{3}^{\text {ext }} \boldsymbol{e}_{3}$, consequently, for a single particle 


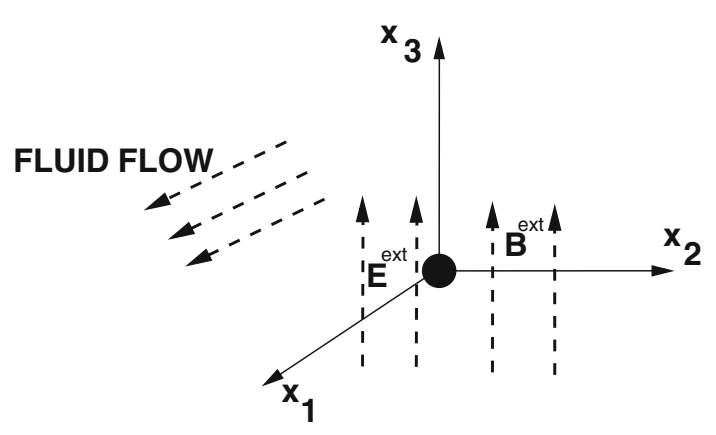

Fig. 1 An isolated particle with an applied "dead" electromagnetic field and fluid-induced drag. For the response, see Fig. 2

$\left\{\begin{array}{l}v_{1}(t) \\ v_{2}(t) \\ v_{3}(t)\end{array}\right\}=\left\{\begin{array}{c}v_{o} \cos \omega t \\ -v_{o} \sin \omega t \\ \frac{q}{m} E_{3}^{\text {ext }} t\end{array}\right\} \Rightarrow\left\{\begin{array}{l}r_{1}(t) \\ r_{2}(t) \\ r_{3}(t)\end{array}\right\}=\left\{\begin{array}{c}\frac{v_{o}}{\omega} \sin \omega t \\ \frac{v_{o}}{\omega}(\cos \omega t-1) \\ \frac{q}{2 m} E_{3}^{\text {ext }} t^{2}\end{array}\right\}$.

where $\omega=\frac{q B_{3}^{e x t}}{m}$ is known as the cyclotron frequency. The cyclotron frequency (gyrofrequency) is the angular frequency at which a charged particle makes circular orbits in a plane perpendicular to the static magnetic field. Notice that when $E_{3}^{\text {ext }}=0$, this traces out the equation of a circle centered at $\left(0,-\frac{v_{o}}{\omega}\right)$. The radius of the "magnetically-induced circle" (radius of oscillation) is ${ }^{3}$

$R \stackrel{\text { def }}{=} \frac{v_{o}}{\omega}=\frac{v_{o} m}{q B_{3}^{\text {ext }}}$.

Thus, if a desired "turning radius" is denoted by $R$, one may solve for the magnetic field that delivers the desired effect, $B_{3}^{\text {ext }}=\frac{v_{o} m}{q R}$. We define the corresponding time period for one cycle to be completed as $T \stackrel{\text { def }}{=} 2 \pi / \omega$. The composite motion is that of a helix, since the third position component grows quadratically in time, while the other two components trace out a circle.

Remark 1 In the special case when there is no magnetic field, if $\boldsymbol{r}(t=0)=\mathbf{0}, \boldsymbol{v}(t=0)=v_{o} \boldsymbol{e}_{1}, \boldsymbol{B}^{\text {ext }}=\mathbf{0}$ and $\boldsymbol{E}^{\text {ext }}=$ $E_{3}^{e x t} e_{3}$, the solution for the dynamics of an isolated particle is

$$
\left\{\begin{array}{l}
v_{1}(t) \\
v_{2}(t) \\
v_{3}(t)
\end{array}\right\}=\left\{\begin{array}{c}
v_{o} \\
0 \\
\frac{q}{m} E_{3}^{\text {ext }} t
\end{array}\right\} \Rightarrow\left\{\begin{array}{l}
r_{1}(t) \\
r_{2}(t) \\
r_{3}(t)
\end{array}\right\}=\left\{\begin{array}{c}
v_{o} t \\
0 \\
\frac{q}{2 m} E_{3}^{\text {ext }} t^{2}
\end{array}\right\} .
$$

Thus, the third position component grows quadratically in time.

Remark 2 In the special case with no electric field and a magnetic field present, $\boldsymbol{r}(t=0)=\mathbf{0}, \boldsymbol{v}(t=0)=v_{o} \boldsymbol{e}_{1}, \boldsymbol{B}^{\text {ext }}=$

\footnotetext{
$\overline{3}$ This field generates helical motion in three dimensions when $\boldsymbol{E}^{\text {ext }} \neq$
}

$B_{3}^{e x t} e_{3}$ and $\boldsymbol{E}^{\text {ext }}=\mathbf{0}$. Consequently, for a single particle, the solution is

$\left\{\begin{array}{l}v_{1}(t) \\ v_{2}(t) \\ v_{3}(t)\end{array}\right\}=\left\{\begin{array}{c}v_{o} \cos \omega t \\ -v_{o} \sin \omega t \\ 0\end{array}\right\} \Rightarrow\left\{\begin{array}{l}r_{1}(t) \\ r_{2}(t) \\ r_{3}(t)\end{array}\right\}=\left\{\begin{array}{c}\frac{v_{o}}{\omega} \sin \omega t \\ \frac{v_{o}}{\omega}(\cos \omega t-1) \\ 0\end{array}\right\}$,

Remark 3 From this point forth, we add a subscript $p$ for particle velocities, to delineate between particles and fluids.

\section{Coupled fluid and electromagnetically-driven dynamics}

We will consider a drag force is given by:

$\boldsymbol{\Psi}^{\text {drag }}=C_{D} \frac{1}{2} A \rho_{f}\left\|\boldsymbol{v}_{f}-\boldsymbol{v}_{p}\right\|^{2} \boldsymbol{\tau}$,

where

$\boldsymbol{\tau} \stackrel{\text { def }}{=} \frac{\boldsymbol{v}_{f}-\boldsymbol{v}_{p}}{\left\|\boldsymbol{v}_{f}-\boldsymbol{v}_{p}\right\|}$,

and $A=\pi R^{2}$, where $R$ is the particle radius. In order to determine the relative strengths of the fluid drag force to the electromagnetic forces acting on the particle, consider the following model for an isolated particle:

$$
\begin{aligned}
& m_{p} \dot{\boldsymbol{v}_{p}}=\boldsymbol{\Psi}^{d r a g}+\boldsymbol{\Psi}^{e+m}=C_{D} \frac{1}{2} A \rho_{f}\left\|\boldsymbol{v}_{f}-\boldsymbol{v}_{p}\right\|^{2} \boldsymbol{\tau} \\
& +\bar{q} V\left(\boldsymbol{E}^{e x t}+\boldsymbol{v}_{p} \times \boldsymbol{B}^{e x t}\right),
\end{aligned}
$$

where $\bar{q}$ is the charge per unit volume and $V=\frac{4}{3} \pi R^{3}$. The general ratio is

$$
\begin{aligned}
& \mathcal{L}\left(\boldsymbol{v}_{p}\right) \stackrel{\text { def }}{=} \frac{\left\|\boldsymbol{\Psi}^{e+m}\right\|}{\left\|\boldsymbol{\Psi}^{\text {drag }}\right\|}=\frac{\left\|\bar{q} V\left(\boldsymbol{E}^{e x t}+\boldsymbol{v}_{p} \times \boldsymbol{B}^{\text {ext }}\right)\right\|}{C_{D} \frac{1}{2} A \rho_{f}\left\|\boldsymbol{v}_{f}-\boldsymbol{v}_{p}\right\|^{2}} \\
& =\frac{8|\bar{q}| R}{3 C_{D} \rho_{f}}\left(\frac{\left\|\boldsymbol{E}^{e x t}+\boldsymbol{v}_{p} \times \boldsymbol{B}^{\text {ext }}\right\|}{\left\|\boldsymbol{v}_{f}-\boldsymbol{v}_{p}\right\|^{2}}\right) .
\end{aligned}
$$

Using the Triangle and Cauchy-Schwarz inequalities:

$\mathcal{L}\left(\boldsymbol{v}_{p}\right) \leq \frac{8|\bar{q}| R}{3 C_{D} \rho_{f}}\left(\frac{\left\|\boldsymbol{E}^{e x t}\right\|+\left\|\boldsymbol{v}_{p}\right\|\left\|\boldsymbol{B}^{e x t}\right\|}{\left\|\boldsymbol{v}_{f}-\boldsymbol{v}_{p}\right\|^{2}}\right)$,

which leads to

$$
\left\|\boldsymbol{E}^{e x t}\right\|+\left\|\boldsymbol{v}_{p}\right\|\left\|\boldsymbol{B}^{e x t}\right\| \geq \mathcal{L}\left(\boldsymbol{v}_{p}\right) \frac{3 C_{D} \rho_{f}\left\|\boldsymbol{v}_{f}-\boldsymbol{v}_{p}\right\|^{2}}{8|\bar{q}| R} .
$$

If we set $\mathcal{L}=1$, we obtain an expression for the electromagnetic forces to have parity with the drag force. In the case when the magnetic field is negligible, we have:

$$
\left\|\boldsymbol{E}^{e x t}\right\|=\frac{3 C_{D} \rho_{f}\left\|\boldsymbol{v}_{f}-\boldsymbol{v}_{p}\right\|^{2}}{8|\bar{q}| R} .
$$


Fig. 2 Right $\mathrm{nN}$ fluid drag resulting in helical motion. Left Drag-induced drift and contraction of the helix
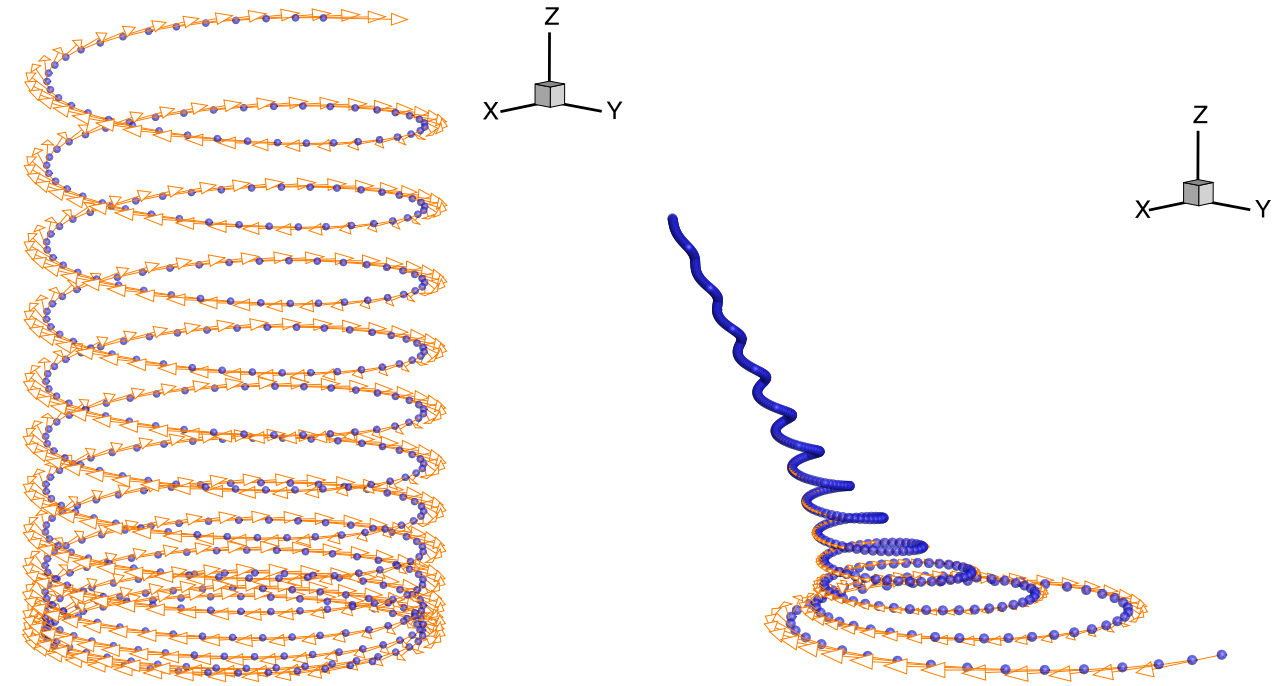

In the case when the electric field is neglible, we have

$$
\left\|\boldsymbol{B}^{e x t}\right\| \geq \frac{3 C_{D} \rho_{f}\left\|\boldsymbol{v}_{f}-\boldsymbol{v}_{p}\right\|^{2}}{8|\bar{q}| R\left\|\boldsymbol{v}_{p}\right\|} \text {. }
$$

These expressions serve as a guide for computationallyintensive multi-particle numerical simulations, which are presented later.

\subsection{Coupled drag-electromagnetically driven dynamics}

Consider the following parameters (in standard SI-units), which are simply chosen just for illustration purposes, and do not correlate directly to any specific physical system:

- $\mu=0.0001$,

- $\rho_{p}=5000$,

- $C_{D}=0.0002$,

- $\boldsymbol{v}_{f}=(0.5,0,0)$,

- $\mathcal{R}=0.001$,

- $\boldsymbol{E}^{\text {ext }}=(0,0,1)$,

- $\boldsymbol{B}^{\text {ext }}=(0,0,1000)$,

- $\rho_{f}=1000$,

- $\bar{q}=1$,

- $\boldsymbol{v}_{p}(t=0)=(1,0,0)$,

- $\boldsymbol{r}_{p}(t=0)=(0,0,0)$.

For such a simple, single-particle system with an independent specified fluid velocity, we can use a simple forwardEuler time-stepping scheme:

$\boldsymbol{v}(t+\Delta t)=\boldsymbol{v}(t)+\frac{\Delta t}{m_{p}}\left(\boldsymbol{\Psi}^{d r a g}(t)+\Psi^{e+m}(t)\right)$.

An extremely small "overkill" (relative to the total simulation time) time-step size of $\Delta t=0.0001$ seconds was used. Further reductions of the time-step size produced no noticeable changes in the results, thus the solutions generated can be considered to have neglibile numerical error. As illustrated in the previous section, analytically, if no fluid was present, a helix would occur (Fig. 2). For these parameter choices, the ratio of electromagnetic forces to that of the fluid were

$\mathcal{L}\left(\boldsymbol{v}_{p}=0.5 \boldsymbol{v}_{f}\right)=26.88$.

thus, a helix that will be gradually dragged (drifts) in the $x_{1}$ (x) direction with the fluid was expected (Fig. 2). Initially, the particle moves in the $x_{1}$ direction which, because of the term $\boldsymbol{v} \times \boldsymbol{B}$, produces a turn to the minus $x_{2}$ (y) direction, which subsequently induces a turn in the $x_{1}$ direction, etc, etc. The fluid dissipates the helical motion and drags it in the $x_{1}$ direction. In reality, the dynamics of the fluid is affected by the particle. Furthermore, multiple particles will lead to possible collisions and further coupling through the fluid. This will be treated numerically, which is the subject of the next few sections.

Remark 1 Qualitatively, one can expect a drag-induced drift in the particle trajectory. For example, consider an isolated particle, moving in one-dimension, with no electromagnetic fields present, in a surrounding fluid in the Stokesian regime, governed by

$m \dot{v}_{p}=\underbrace{c\left(v_{f}-v_{p}\right)}_{\text {drag }}$,

which yields

$v_{p}(t)=\left(v_{p}(0)-v_{f}\right) e^{-c t / m}+v_{f}$.

In the special case where $m=\rho_{p} \frac{4}{3} \pi R^{3}$ and the Stokesian drag is $c=c_{o} 6 \pi R$. 
$\dot{v}_{p}=\underbrace{\frac{9 c_{o}}{2 \rho_{p} \mathcal{R}^{2}}}_{\text {drag } \stackrel{\text { def }}{=} \alpha}\left(v_{f}-v_{p}\right)$,

the velocity can be determined to be

$v_{p}(t)=\left(v_{p}(0)-v_{f}\right) e^{-\alpha t}+v_{f}$.

Thus, qualitatively, we expect such a fluid-induced drift to occur.

Remark 2 Another class of electromagnetically sensitive fluids are Ferrofluids, which are colloidal liquids of ferromagnetic nanoscale (10 nanometers or less) particles, often from a iron-based compound, such as magnetite or hematite, usually in an organic solvent. The volume fraction of the particles is typically under 5 percent by volume. The particles are usually coated with a surfactant (typically oelic acid, citric acid, soy lecithin, tetramethylammonium hydroxide) to avoid agglomeration. The surfactant is strong enough to counteract near-field interaction effects between particles. The particles usually do not retain magnetization and can be considered as paramagnetic. Furthermore, they lose their magnetic properties at sufficiently high (Curie) temperatures. ${ }^{4}$ We refer the reader to Albrecht et al. [1], Andelman and Rosensweig [3] and Berger et al. [5] for reviews. If the particles exhibit magnetization, an additional force is exerted, independent of the electrodynamically-induced Lorentz forces. A simple model to characterize this force is given by

$\boldsymbol{\Psi}^{\text {mag }}=-\nabla\left(\gamma \boldsymbol{B}^{\text {ext }} \cdot \boldsymbol{B}^{\text {ext }}\right)$,

where $\gamma$ is a material parameter that is related to the magnetic dipole properties, the magnetization of the particle, and which is dependent on the magnetic susceptibility, the magnetic permeability and the internal magnetic moment density of the material (see Feynman et al. [9], Cullity and Graham [8], Boyer [6] or Jackson [17]. We note that while we will not simulate purely magnetic (or magnetizable) particles in numerical examples presented later, we include the relevant terms in the formulation.

\section{Numerical simulation of coupled fluid-multi-particle systems}

There are three components to the numerical discretization of the coupled fluid-particle system:

- Temporal discretzation of the particle dynamics model,

- Spatio-temporal discretization of the fluid continuum model and

\footnotetext{
${ }^{4}$ Another class of elecromagnetically sensitive particle laden fluid are magnetorheological fluids which are seeded with micron-sized particles, and consequently the particles can sediment over time.
}

- Iterative staggering scheme to resolve the coupling between the particles and fluid continuum.

\subsection{The overall approach}

The present section develops a flexible and robust solution strategy to resolve two-way coupled systems comprised of large groups of flowing particles embedded within a fluid. Such particle/fluid systems are strongly coupled due to the forces induced by the fluid onto the particles and vice-versa. Specifically, a staggered approach is developed whereby, at each time-step:

- A pure particle-only system is solved where the fluid's influence is accounted for by drag forces on the particles.

- A pure fluid-only system is then solved where the particles' influence is accounted for by reverse drag forces on the fluid continuum.

- The interactions between the particles and fluid are then updated. Within a time-step, this process is repeated until the system converges. If the system does not converge within a preset number of iterations, the time-steps are reduced by a prescribed amount, dictated by an estimate of the spectral radius of the coupled system.

Because the coupling of the various particle and fluid fields can dramatically change over the course of a flow process, a primary focus is the development of a recursive "staggering" solution scheme, whereby the time-steps are adaptively adjusted to control the error associated with the incomplete resolution of the coupled interaction between the various solid particulate and continuum fluid fields.

\subsection{Simplifying assumptions}

We make the following simplifying assumptions:

- We assume that each particle is small enough that their rotation with respect to their mass centers is deemed insignificant. The particles are considered to be spheres.

- We assume that any pre-applied particle coatings/surfactants are thin enough to be considered as part of the particle.

- We assume the external electromagnetic fields are given, and that action of the particle-laden fluid does not affect the fields-i.e. they are considered control variables.

- We assume that the fluid is incompressible.

Other simplifying assumptions will be made later in the presentation where appropriate. 


\section{Modeling and simulation of the particle dynamics problem}

\subsection{Direct particle representation}

We consider a group of non-intersecting particles $(i=$ $\left.1,2, \ldots, N_{p}\right)$. The equation of motion for the $i t h$ particle in the system is

$$
\begin{aligned}
m_{i} \dot{\boldsymbol{v}}_{p i} & =m_{i} \ddot{\boldsymbol{r}}_{p i}=\boldsymbol{\Psi}_{i}^{\text {tot }}\left(\boldsymbol{r}_{p 1}, \boldsymbol{r}_{p 2}, \ldots, \boldsymbol{r}_{p N_{p}}\right) \\
& =\boldsymbol{\Psi}_{i}^{\text {con }}+\boldsymbol{\Psi}_{i}^{\text {drag }}+\boldsymbol{\Psi}_{i}^{e+m},
\end{aligned}
$$

where $\boldsymbol{r}_{p i}$ is the position vector of the $i t h$ particle and where $\boldsymbol{\Psi}_{i}^{t o t}$ represents all forces acting on particle $i$, which is decomposed into the sum of forces due to the normal and frictional contact forces $\left(\boldsymbol{\Psi}_{i}^{\text {con }}\right)$, the drag forces arising from the surrounding fluid environment $\left(\boldsymbol{\Psi}_{i}^{\text {drag }}\right)$ and electromagnetic forces $\left(\boldsymbol{\Psi}_{i}^{e+m}\right)$. In the next sections, we discuss of each of the types of forces in the system in detail.

\subsection{Particle-to-particle contact forces}

For completeness, we include particle-to-particle contact, which technically requires breaking the fluid film between the particles. One can consider this type of interaction as an "effective" contact model. We employ a simple particle overlap model to determine the force contributions from the surrounding particles $\left(N_{c i}\right)$ in contact, $\Psi_{i}^{c o n, n}=\sum_{j=1}^{N_{c i}} \boldsymbol{\psi}_{i j}^{c o n, n}$, based on a normalized separation distance between particles in contact (Figs. 3, 4). We assume that the contact force is proportional to the relative normalized proximity of the particles. For example, a simple relation for particles in contact $\left(\left\|\boldsymbol{r}_{p i}-\boldsymbol{r}_{p j}\right\| \leq R_{i}+R_{j}\right)$ is

$\boldsymbol{\Psi}_{i j}^{c o n, n} \propto-K^{p}\left|\mathcal{E}_{i j}\right|^{p_{p}} \boldsymbol{n}_{i j} A_{i j}^{c}$,

where $0<K^{p}<\infty$ is a particle-to-particle contact compliance constant, $A_{i j}^{c}$ is a contact area parameter (derived in the Appendix), $p_{p}$ is a material parameter, $\mathcal{E}_{i j}$ is normalized/nondimensional (strain-like) deformation metric

$\mathcal{E}_{i j}=\frac{\left\|\boldsymbol{r}_{p i}-\boldsymbol{r}_{p j}\right\|-\left(R_{i}+R_{j}\right)}{\left(R_{i}+R_{j}\right)}$

and

$\boldsymbol{n}_{i j}=-\frac{\boldsymbol{r}_{p i}-\boldsymbol{r}_{p j}}{\left\|\boldsymbol{r}_{p i}-\boldsymbol{r}_{p j}\right\|}=\frac{\boldsymbol{r}_{p j}-\boldsymbol{r}_{p i}}{\left\|\boldsymbol{r}_{p i}-\boldsymbol{r}_{p j}\right\|}$,

where the $R_{i}$ and $R_{j}$ are the radii of particles $i$ and $j$ respectively.

\subsection{Stick/slip}

Frictional stick is modelled via the following regularized friction algorithm:
- Check static friction threshold: $\left(K^{f}\right.$ is a frictional contact compliance)

$$
\left\|K^{f}\left(\boldsymbol{v}_{p i, t}-\boldsymbol{v}_{p j, t}\right) \Delta t\right\| \text { against } \mu_{s}\left\|\Psi^{c o n, n}\right\| .
$$

- If the threshold is not exceeded, then:

$$
\boldsymbol{\Psi}^{c o n, f}=K^{f}\left(\boldsymbol{v}_{p i, \tau}-\boldsymbol{v}_{p j, \tau}\right) \Delta t \boldsymbol{\tau}_{i j}
$$

where

$$
\boldsymbol{\tau}_{i j}=-\frac{\boldsymbol{v}_{p i, \tau}-\boldsymbol{v}_{p j, \tau}}{\left\|\boldsymbol{v}_{p j, \tau}-\boldsymbol{v}_{p i, \tau}\right\|}=\frac{\boldsymbol{v}_{p j, \tau}-\boldsymbol{v}_{p i, \tau}}{\left\|\boldsymbol{v}_{p j, \tau}-\boldsymbol{v}_{p i, \tau}\right\|}
$$

and where the subscripts " $\tau$ " indicate tangential components.

- If the threshold is met, or exceeded, then enact slip. For the generated friction, we assume a simple contact-induced sliding friction relation:

$$
\boldsymbol{\Psi}_{i j}^{c o n, f}=\mu_{d}\left\|\boldsymbol{\Psi}_{i j}^{c o n, n}\right\| \boldsymbol{\tau}_{i j}
$$

Remark One can easily construct more elaborate relations connecting the relative proximity of the particles and other metrics to the contact force, $\Psi_{i j}^{c o n, n} \propto \mathcal{F}\left(\boldsymbol{r}_{p i}, \boldsymbol{r}_{p j}, \boldsymbol{n}_{i j}\right.$, $R_{i}, R_{j}, \ldots$ ), for example building on Hertzian contact models. This poses no difficulty in the direct numerical method developed. For the remainder of the analysis, we shall use the deformation metric in Eq. 5.3. The Appendix provides classical alternatives provided by Hertzian models. For detailed treatments, see Wellman et al. [40-44] and Avci and Wriggers [4].

\subsection{Electromagnetic forces}

The electromagnetic forces are decomposed into three contributions, (1) Lorentz forces (for charged particles), (2) magnetic forces (for magnetic particles) and (3) inter-particle near-field forces. We will utilize the decomposition:

$$
\begin{aligned}
\boldsymbol{\Psi}_{i}^{e+m} & =\boldsymbol{\Psi}_{i}^{\text {lor,e+m }}+\boldsymbol{\Psi}_{i}^{m a g}+\underbrace{\sum_{j \neq i}^{N} \boldsymbol{\psi}_{i j}^{n f}}_{\boldsymbol{\Psi}_{i}^{n f}} \\
& =\underbrace{q_{i}\left(\boldsymbol{E}^{\text {ext }}+\boldsymbol{v}_{p i} \times \boldsymbol{B}^{\text {ext }}\right)}_{\boldsymbol{\Psi}^{\text {lor }, e+m_{i}}}+\boldsymbol{\Psi}_{i}^{\text {mag }}+\boldsymbol{\Psi}_{i}^{n f},
\end{aligned}
$$

where $\sum_{j \neq i}^{N} \psi_{i j}^{n f}$ represents the interaction between particle $i$ and all other particles $j=1,2, \ldots, N(j \neq i)$, $\boldsymbol{\Psi}_{i}^{\text {lor }, e+m}$ represents external Lorentz-induced forces from the surrounding environment, for example comprised of $\boldsymbol{E}^{\text {ext }}$ and $\boldsymbol{B}^{\text {ext }}$, which are externally-controlled fields that are independent of the response of the system. $\boldsymbol{E}^{e x t}$ and $\boldsymbol{B}^{e x t}$ can be considered as static (or extremely slowly-varying), and thus 
Fig. 3 Decomposition of the coupled fluid-system

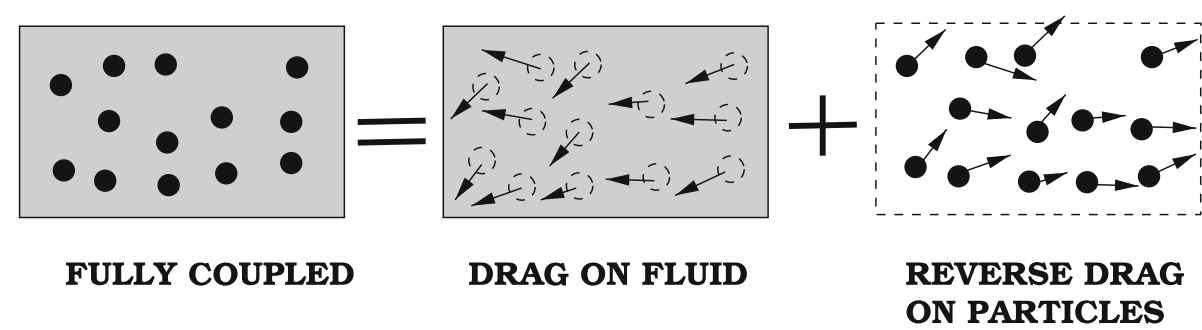

mutually uncoupled and independently controllable. For the specific embedded particle-fluid applications in this paper, $\boldsymbol{\Psi}_{i}^{\text {mag }}$ and $\boldsymbol{\Psi}_{i}^{n f}$ are considered small ${ }^{5}$. For more details on the near-field forces, see the Appendix.

\subsection{Total forces acting on particles}

In summary, we have the following forces acting on each particle $\left(i=1,2, \ldots, N_{p}\right)^{6}$

$\boldsymbol{\Psi}_{i}^{t o t} \stackrel{\text { def }}{=} \boldsymbol{\Psi}_{i}^{c o n}+\boldsymbol{\Psi}_{i}^{d r a g}+\boldsymbol{\Psi}_{i}^{e+m}$

Integrating Equation 5.1 leads to (using a trapezoidal rule with variable integration metric, $0 \leq \phi \leq 1)$

$$
\begin{aligned}
\boldsymbol{v}_{p i}(t+\Delta t) & =\boldsymbol{v}_{p i}(t)+\frac{1}{m_{i}} \int_{t}^{t+\Delta t} \boldsymbol{\Psi}_{i}^{t o t} d t \\
& \approx \boldsymbol{v}_{p i}(t)+\frac{\Delta t}{m_{i}}\left(\phi \Psi_{i}^{t o t}(t+\Delta t)+(1-\phi) \Psi_{i}^{t o t}(t)\right) .
\end{aligned}
$$

The position can be computed via application of the trapezoidal rule again:

$\boldsymbol{r}_{p i}(t+\Delta t) \approx \boldsymbol{r}_{p i}(t)+\Delta t\left(\phi \boldsymbol{v}_{p i}(t+\Delta t)+(1-\phi) \boldsymbol{v}_{p i}(t)\right)$,

which can be consolidated into

$$
\begin{aligned}
& \boldsymbol{r}_{p i}(t+\Delta t)=\boldsymbol{r}_{p i}(t)+\boldsymbol{v}_{p i}(t) \Delta t \\
& \quad+\frac{\phi(\Delta t)^{2}}{m_{i}}\left(\phi \boldsymbol{\Psi}_{i}^{t o t}(t+\Delta t)+(1-\phi) \Psi_{i}^{t o t}(t)\right) .
\end{aligned}
$$

This leads to a coupled system of equations, which are solved using an adaptive iterative scheme, building on approaches found in various forms in Zohdi [50,51,55,56,58-62]. This is dealt with after introducing the governing fluid mechanics equations.

\footnotetext{
${ }^{5}$ Furthermore, the self-induced magnetic fields developed between particles are insignificant for the velocity ranges of interest here (well below the speed of light).

${ }^{6}$ For completeness, we have included an additional force can exerted on magnetic particles.
}

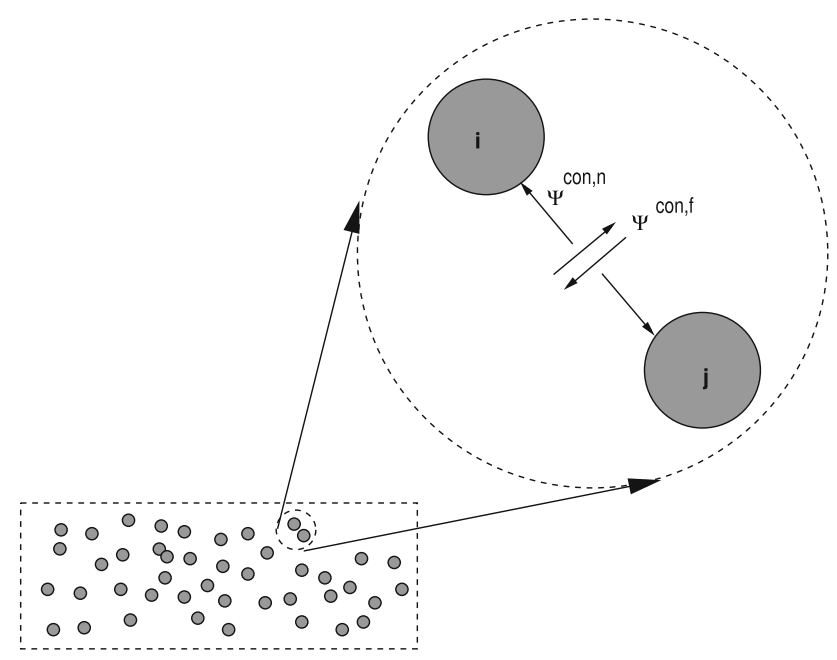

Fig. 4 Normal contact and friction forces

\section{Characterization of particle/fluid interaction}

We first consider drag-force interactions between the fluid and particles. The Appendix provides a concise review of the governing fluids equations. The drag-force acting on an object in a fluid flow (occupying domain $\Omega$ and outward surface normal $\boldsymbol{n}$ ) is defined as

$\boldsymbol{\Psi}^{d r a g}=\int_{\partial \Omega} \boldsymbol{\sigma} \cdot \boldsymbol{n} d A$

where $\sigma$ is the Cauchy-stress. For a Newtonian fluid obeying the Stokes' condition has the following constitutive law (see Appendix):

$\boldsymbol{\sigma}=-P \mathbf{1}-\frac{2}{3} \mu t r \boldsymbol{D} \mathbf{1}+2 \mu \boldsymbol{D}=-P \mathbf{1}+2 \mu \boldsymbol{D}^{\prime}$.

where $P$ is the thermodynamic pressure, $\mu$ is the absolute viscosity, $\boldsymbol{D}=\frac{1}{2}\left(\nabla_{\boldsymbol{x}} \boldsymbol{v}_{f}+\left(\nabla_{\boldsymbol{x}} \boldsymbol{v}_{f}\right)^{T}\right)$ is the symmetric part of the velocity gradient, $\operatorname{tr} \boldsymbol{D}$ is the trace of $\boldsymbol{D}$, and where $\boldsymbol{D}^{\prime}=$ $\boldsymbol{D}-\frac{\operatorname{tr} \boldsymbol{D}}{3} \mathbf{1}$ is the deviatoric part of $\boldsymbol{D}$. The stress is determined by solving the balance of linear momentum (Navier-Stokes)

$\rho_{f}\left(\frac{\partial \boldsymbol{v}_{f}}{\partial t}+\left(\nabla_{\boldsymbol{x}} \boldsymbol{v}_{f}\right) \cdot \boldsymbol{v}_{f}\right)=\nabla_{\boldsymbol{x}} \cdot \boldsymbol{\sigma}+\boldsymbol{f}_{f}$, 
where, at a point, $\rho_{f}$ is the fluid density, $\boldsymbol{v}_{f}$ is the fluid velocity, and where $\boldsymbol{f}_{f}$ represents body forces per unit voulme. We refer the reader to the Appendix for more details on the fluid model.

The fluid domain will require spatial discretization with some type of mesh, for example using a finite difference, finite volume or finite element method. Usually, it is extremely difficult to resolve the flow in the immediate neighborhood of the particles, in particular if there are several particles. However, if the primary interest is in the dynamics of the particles, as it is in this work, an appropriate approach, which permits coarser discretization of the fluid continuum, is to employ effective drag coefficients, for example, as introduced earlier ${ }^{7}$

$C_{D} \stackrel{\text { def }}{=} \frac{\left\|\Psi_{i}^{d r a g}\right\|}{\frac{1}{2} \rho_{f}\left\|\left\langle\boldsymbol{v}_{f}\right\rangle_{\Omega_{i}}-\boldsymbol{v}_{p i}\right\|^{2} A_{i}}$,

where $\langle(\cdot)\rangle_{\Omega_{i}} \stackrel{\text { def }}{=} \frac{1}{\left|\Omega_{i}\right|} \int_{\Omega_{i}}(\cdot) d \Omega_{i}$ is the volumetric average of the argument over the domain occupied by the ith particle, $\left\langle\boldsymbol{v}_{f}\right\rangle_{\Omega_{i}}$ is the volumetric average of the fluid velocity, where $A_{i}$ is the cross-sectional area of the $i t h$ (solid) particle. The use of this simple concept is relatively straightforward to account for the presence of the solid particles in the fluid by augmenting the flow calculations with drag-forces (Fig. 3). Algorithmically speaking, one must compute the fluid flow with reaction forces due to the presence of the particles. To this end, one can use the volumetric forces $\left(f_{f}\right)$ within the fluid domain for this purpose by writing

$$
\begin{aligned}
\boldsymbol{f}_{f}= & -\frac{\boldsymbol{\Psi}_{p i}^{\text {drag }}}{\left|\Omega_{p i}^{*}\right|}=-\frac{C_{D} \frac{1}{2} \rho_{f}\left\|\left\langle\boldsymbol{v}_{f}\right\rangle_{\Omega_{p i}^{*}}-\boldsymbol{v}_{p i}\right\|^{2} A_{i}}{\left|\Omega_{p i}^{*}\right|} \hat{\boldsymbol{\tau}} \\
& \times\left(\hat{\boldsymbol{\tau}}=\frac{\left\langle\boldsymbol{v}_{f}\right\rangle_{\Omega_{p i}^{*}}-\boldsymbol{v}_{p i}}{\left\|\left\langle\boldsymbol{v}_{f}\right\rangle_{\Omega_{p i}^{*}}-\boldsymbol{v} p i\right\|}\right)
\end{aligned}
$$

where $\left|\Omega_{p i}^{*}\right|$ is the equivalent volume of the number of nodes (each node with assigned volume $\Delta x_{1} \Delta x_{2} \Delta x_{3}$ ) that fall within the particle domain and $\boldsymbol{f}_{f}$ (per unit volume) is the drag-force on the fluid, which is nonzero if a node falls within the particle domain, and is zero otherwise. ${ }^{8}$ This drag-based approach is designed to account for particles in the fluid using a coarse mesh. In summary, the drag force is computed by calculating the difference between the average fluid

\footnotetext{
${ }^{7}$ In the general compressible case one would use

$\left\langle\rho_{f i}\right\rangle_{\Omega_{p i}} \stackrel{\text { def }}{=} \frac{1}{\Omega_{p i}} \int_{\Omega_{p i}} \rho(\boldsymbol{x}) d \Omega$,

instead of $\rho_{f}$.

${ }^{8}$ If the particles are significantly smaller than the mesh spacing, then the drag-forces associated with the particles are computed from the nearest node/particle center pair.
}

velocity in the $i t h$ particle domain $\left\langle\boldsymbol{v}_{f i}\right\rangle_{\Omega_{p i}}$ and the particle velocity (no particle rotation is assumed), $\boldsymbol{v}_{p i}$ to yield the drag force $\boldsymbol{\Psi}_{i}^{\text {drag }} \stackrel{\text { def }}{=} \frac{1}{2} C_{D} \rho_{f}\left\|\left\langle\boldsymbol{v}_{f i}\right\rangle_{\Omega_{p i}}-\boldsymbol{v}_{p i}\right\|^{2} A_{i} \hat{\boldsymbol{\tau}}$.

Remark 1 From this point forward, we will consider incompressible cases ( $\rho_{f}$ is constant) and where $P$ is given. The Appendix provides a more general formulation.

Remark 2 There are a variety of empirical relations for the drag coefficient. One possible way to represent the drag coefficient is with a piecewise definition, as a function of the Reynolds number (Chow [7]):

- For $0<R e \leq 1, C_{D}=\frac{24}{R e}$,

- For $1<R e \leq 400, C_{D}=\frac{24}{R e^{0.646}}$,

- For $400<R e \leq 3 \times 10^{5}, C_{D}=0.5$,

- For $3 \times 10^{5}<R e \leq 2 \times 10^{6}, C_{D}=0.000366 R e^{0.4275}$,

- For $2 \times 10^{6}<\operatorname{Re}<\infty, C_{D}=0.18$,

where the local Reynolds number for a particle is $R e \stackrel{\text { def }}{=}$ $\frac{2 R_{i} \rho_{f}\left\|\left\langle\boldsymbol{v}_{f}\right\rangle_{\Omega_{i}}-\boldsymbol{v}_{p i}\right\|}{\mu_{f}}$ and where $\mathcal{R}_{i}$ is the radius of the $i t h$ particle.

\section{Discretization of the fluid}

\subsection{Temporal discretization}

For the fluid, we write

$\frac{d \boldsymbol{v}_{f}}{d t}=\frac{\partial \boldsymbol{v}_{f}}{\partial t}+\nabla_{x} \boldsymbol{v}_{f} \cdot \boldsymbol{v}_{f}=\frac{1}{\rho_{f}}\left(\nabla_{x} \cdot \boldsymbol{\sigma}+\boldsymbol{f}\right)$,

leading to

$\frac{\partial \boldsymbol{v}_{f}}{\partial t}=\frac{1}{\rho_{f}}\left(\nabla_{x} \cdot \boldsymbol{\sigma}+\boldsymbol{f}\right)-\nabla_{x} \boldsymbol{v}_{f} \cdot \boldsymbol{v}_{f} \stackrel{\text { def }}{=} \boldsymbol{L}$.

We discretize for time $=t+\phi \Delta t$, and using a trapezoidal " $\phi-$ scheme" $(0 \leq \phi \leq 1)$

$$
\begin{aligned}
& \frac{\partial \boldsymbol{v}_{f}}{\partial t} \approx \frac{\boldsymbol{v}_{f}(t+\Delta t)-\boldsymbol{v}_{f}(t)}{\Delta t} \approx \boldsymbol{L}(t+\phi \Delta t) \approx \phi \boldsymbol{L}(t+\Delta t) \\
& \quad+(1-\phi) \boldsymbol{L}(t) .
\end{aligned}
$$

Rearranging yields

$$
\boldsymbol{v}_{f}(t+\Delta t) \approx \boldsymbol{v}_{f}(t)+\Delta t(\phi \boldsymbol{L}(t+\Delta t)+(1-\phi) \boldsymbol{L}(t))
$$

where the previously introduced spatial discretization is applied to the derivative terms (such as $\nabla_{x} \cdot \boldsymbol{\sigma}$ ) in $\boldsymbol{L}$. The discretized system is formulated next as an implicit timestepping scheme within each time step $L$. 
7.2 Spatial discretization: spatial finite difference stencils

The following standard approximations are used:

1. For the first derivative of a primal variable $v$ at $\left(x_{1}, x_{2}, x_{3}\right)$ :

$$
\frac{\partial v_{f}}{\partial x_{1}} \approx \frac{v_{f}\left(x_{1}+\Delta x_{1}, x_{2}, x_{3}\right)-v_{f}\left(x_{1}-\Delta x_{1}, x_{2}, x_{3}\right)}{2 \Delta x_{1}}
$$

2. For the derivative of a flux at $\left(x_{1}, x_{2}, x_{3}\right)$ :

$$
\begin{aligned}
\frac{\partial}{\partial x_{1}}\left(A \frac{\partial v_{f}}{\partial x_{1}}\right) & \\
\approx & \frac{\left.\left(A \frac{\partial v_{f}}{\partial x_{1}}\right)\right|_{x_{1}+\frac{\Delta x_{1}}{2}, x_{2}, x_{3}}-\left.\left(A \frac{\partial v_{f}}{\partial x_{1}}\right)\right|_{x_{1}-\frac{\Delta x_{1}}{2}, x_{2}, x_{3}}}{\Delta x_{1}} \\
= & \frac{1}{\Delta x_{1}}\left[A\left(x_{1}+\frac{\Delta x_{1}}{2}, x_{2}, x_{3}\right)\right. \\
- & \frac{1}{\Delta x_{1}}\left[A\left(x_{1}-\frac{\Delta x_{1}}{2}, x_{2}, x_{3}\right)\right. \\
& \left.\left(\frac{v_{f}\left(x_{1}, x_{2}, x_{3}\right)-x_{f}\left(x_{1}-\Delta x_{1}, x_{2}, x_{3}\right)}{\Delta x_{1}}\right)\right],
\end{aligned}
$$

where we have used

$$
\begin{gathered}
A\left(x_{1}+\frac{\Delta x_{1}}{2}, x_{2}, x_{3}\right) \approx \frac{1}{2}\left(A\left(x_{1}+\Delta x_{1}, x_{2}, x_{3}\right)\right. \\
\left.+A\left(x_{1}, x_{2}, x_{3}\right)\right)
\end{gathered}
$$

and

$$
\begin{gathered}
A\left(x_{1}-\frac{\Delta x_{1}}{2}, x_{2}, x_{3}\right) \approx \frac{1}{2}\left(A\left(x_{1}, x_{2}, x_{3}\right)\right. \\
\left.+A\left(x_{1}-\Delta x_{1}, x_{2}, x_{3}\right)\right)
\end{gathered}
$$

3. For the cross-derivative of a flux at $\left(x_{1}, x_{2}\right)$ :

$$
\begin{aligned}
\frac{\partial}{\partial x_{2}}\left(A \frac{\partial v_{f}}{\partial x_{1}}\right) & \\
\approx & \frac{\partial}{\partial x_{2}}\left(A\left(x_{1}, x_{2}, x_{3}\right)\right. \\
& \left.\left.\left(\frac{v_{f}\left(x_{1}+\Delta x_{1}, x_{2}, x_{3}\right)-v_{f}\left(x_{1}-\Delta x_{1}, x_{2}, x_{3}\right)}{2 \Delta x_{1}}\right)\right)\right) \\
\approx & \frac{1}{4 \Delta x_{1} \Delta x_{2}}\left(A\left(x_{1}, x_{2}+\Delta x_{2}, x_{3}\right)\right. \\
& {\left[v_{f}\left(x_{1}+\Delta x_{1}, x_{2}+\Delta x_{2}, x_{3}\right)\right.} \\
& \left.-v_{f}\left(x_{1}-\Delta x_{1}, x_{2}+\Delta x_{2}, x_{3}\right)\right] \\
- & A\left(x_{1}, x_{2}-\Delta x_{2}, x_{3}\right)\left[v_{f}\left(x_{1}+\Delta x_{1}, x_{2}-\Delta x_{2}, x_{3}\right)\right. \\
& \left.\left.-v_{f}\left(x_{1}-\Delta x_{1}, x_{2}-\Delta x_{2}, x_{3}\right)\right]\right),
\end{aligned}
$$

7.3 Overall iterative (implicit) solution method

Following the basic framework in Zohdi [50,51,55,56,58$62]$, let us consider the finite difference nodes $(i)$ :

$\boldsymbol{v}_{f i}^{L+1, K}=\boldsymbol{v}_{f i}^{L}+\Delta t\left(\phi \boldsymbol{L}_{i}^{L+1, K-1}+(1-\phi) \boldsymbol{L}_{i}^{L}\right)$,

where $i$ is the node counter, which is of the form

$\boldsymbol{v}_{f i}^{L+1, K}=\mathcal{G}\left(\boldsymbol{v}_{f i}^{L+1, K-1}\right)+R_{i}$,

where $K=1,2,3, \ldots$ is the index of iteration within time step $L+1$ and

- $\mathcal{G}\left(\boldsymbol{v}_{f i}^{L+1, K-1}\right)=\phi \Delta t \boldsymbol{L}_{i}^{L+1, K-1}$ and

- $R_{i}=\boldsymbol{v}_{f i}^{L}+\Delta t(1-\phi) L^{L}$.

The term $R_{i}$ is a remainder term that does not depend on the solution. The convergence of such a scheme is dependent on the behavior of $\mathcal{G}$. Namely, a sufficient condition for convergence is that $\mathcal{G}$ is a contraction mapping for all $\boldsymbol{v}_{f i}^{L+1, K}, K=1,2,3 \ldots$ In order to investigate this further, we define the iteration error as

$\varpi_{i}^{L+1, K} \stackrel{\text { def }}{=} \boldsymbol{v}_{f i}^{L+1, K}-\boldsymbol{v}_{f i}^{L+1}$.

A necessary restriction for convergence is iterative self consistency, i.e. the "exact" (discretized) solution must be represented by the scheme, $v_{i}^{L+1}=\mathcal{G}\left(\boldsymbol{v}_{i}^{L+1}\right)+R_{i}$. Enforcing this restriction, a sufficient condition for convergence is the existence of a contraction mapping

$$
\begin{aligned}
& \|\underbrace{\boldsymbol{v}_{f i}^{L+1, K}-\boldsymbol{v}_{f i}^{L+1}}_{\varpi_{i}^{L+1, K}}\|=\left\|\mathcal{G}\left(\boldsymbol{v}_{f i}^{L+1, K-1}\right)-\mathcal{G}\left(\boldsymbol{v}_{f i}^{L+1}\right)\right\| \\
& \leq \eta^{L+1, K}\left\|\boldsymbol{v}_{i}^{L+1, K-1}-\boldsymbol{v}_{f i}^{L+1}\right\|,
\end{aligned}
$$

where, if $0 \leq \eta^{L+1, K}<1$ for each iteration $K$, then $\bar{\varpi}_{i}^{L+1, K} \rightarrow \overline{\mathbf{0}}$ for any arbitrary starting value $\boldsymbol{r}_{i}^{L+1, K=0}$, as $K \rightarrow \infty$, which is a contraction condition that is sufficient, but not necessary, for convergence. The convergence of Equation 7.10 is scaled by $\eta \propto \frac{(\phi \Delta t)^{2}}{m_{i}}$. Therefore, we see that the contraction constant of $\mathcal{G}$ is:

- directly dependent on the magnitude of the interaction forces $(\|\boldsymbol{L}\|)$,

- directly proportional to $(\Delta t)^{2}$.

Thus, decreasing the time step size improves the convergence. In order to maximize the time-step sizes (to decrease overall computing time) and still meet an error tolerance on the numerical solution's accuracy, we build on an approach 
originally developed for continuum thermo-chemical multifield problems (Zohdi [51]), where one assumes: (1) $\eta^{L+1, K} \approx S(\Delta t)^{p},(S$ is a constant $)$ and (2) the error within an iteration behaves according to $\left(S(\Delta t)^{p}\right)^{K} \varpi^{L+1,0}=$ $\varpi^{L+1, K}, K=1,2, \ldots$, where $\varpi^{L+1,0}=\boldsymbol{v}_{f i}^{L+1, K=1}-\boldsymbol{v}_{f i}^{L}$ is the initial norm of the iterative (relative) error and $S$ is intrinsic to the system. For example, for second-order problems, due to the quadratic dependency on $\Delta t, p \approx 2$. The objective is to meet an error tolerance in exactly a preset (the analyst sets this) number of iterations. To this end, one writes $\left(S\left(\Delta t_{\mathrm{tol}}\right)^{p}\right)^{K_{d}} \varpi^{L+1,0}=T O L$, where $T O L$ is a tolerance and where $K_{d}$ is the number of desired iterations. If the error tolerance is not met in the desired number of iterations, the contraction constant $\eta^{L+1, K}$ is too large. Accordingly, one can solve for a new smaller step size, under the assumption that $S$ is constant,

$$
\Delta t_{\mathrm{tol}}=\Delta t \underbrace{\left(\frac{\left(\frac{T O L}{\varpi^{L+1,0}}\right)^{\frac{1}{p K}}}{\left(\frac{\varpi^{L+1, K}}{\varpi^{L+1,0}}\right)^{\frac{1}{p K}}}\right)}_{\stackrel{\text { def }}{=} \Lambda_{K}} .
$$

The assumption that $S$ is constant is not critical, since the time steps are to be recursively refined and unrefined throughout the simulation. Clearly, the expression in Eq. 7.14 can also be used for time step enlargement, if convergence is met in less than $K_{d}$ iterations (typically chosen to be between five to ten iterations). Specifically, the solution steps are, within a time-step:

- (1): Start a global fixed iteration ( set $i=1, \ldots, N_{n}$ (node counter) and $K=0$ (iteration counter))

- (2): If $i>N_{n}$ then go to (4)

- (3): If $i \leq N_{n}$ then:

(a) Compute the velocity $\boldsymbol{v}_{f i}^{L+1, K}$

(b) Go to (2) for the next node $(i=i+1)$

- (4): Repeat steps 1-3 for the particles, $i=1, \ldots, N_{p}$ and compute the drag forces.

- (5): Measure error (normalized) quantities (where $w_{f}$ is a weight on the fluid contribution and $w_{p}$ is a weight on the particle contribution)

(a) $\varpi_{K} \stackrel{\text { def }}{=} w_{f} \frac{\sum_{i=1}^{N_{n}}\left\|\boldsymbol{v}_{f i}^{L+1, K}-\boldsymbol{v}_{f i}^{L+1, K-1}\right\|}{\sum_{i=1}^{N_{n}}\left\|\boldsymbol{v}_{f i}^{L+1, K}\right\|}$

$$
+w_{p} \frac{\sum_{i=1}^{N_{p}}\left\|\boldsymbol{v}_{p i}^{L+1, K}-\boldsymbol{v}_{p i}^{L+1, K-1}\right\|}{\sum_{i=1}^{N_{p}}\left\|\boldsymbol{v}_{p i}^{L+1, K}\right\|}
$$

(b) $Z_{K} \stackrel{\text { def }}{=} \frac{\varpi_{K}}{T O L_{r}}$

(c) $\Lambda_{K} \stackrel{\text { def }}{=}\left(\frac{\left(\frac{T O L}{\varpi_{0}}\right)^{\frac{1}{p K_{d}}}}{\left(\frac{\varpi_{K}}{\varpi_{0}}\right)^{\frac{1}{p K}}}\right)$.
Note As an option, one could select to only use the error metric from the Finite Difference grid, since the particle velocities have already been projected onto the grid.

- (6): If the tolerance is met: $Z_{K} \leq 1$ and $K<K_{d}$ then

(a) Increment time: $t=t+\Delta t$

(b) Construct the next time step: $(\Delta t)^{\text {new }}=\Lambda_{K}(\Delta t)^{\text {old }}$,

(c) Select the minimum size: $\Delta t=\operatorname{MIN}\left((\Delta t)^{\lim }\right.$, $(\Delta t)^{\text {new }}$ ) and go to (1)

- (7): If the tolerance is not met: $Z_{K}>1$ and $K<K_{d}$ then

(a) Update the iteration counter: $K=K+1$

(b) Reset the node counter: $i=1$

(c) Go to (2)

- (8): If the tolerance is not met $\left(Z_{K}>1\right)$ and $K=K_{d}$ then

(a) Construct a new time step: $(\Delta t)^{\text {new }}=\Lambda_{K}(\Delta t)^{\text {old }}$

(b) Restart at time $t$ and go to (1)

Time-step size adaptivity is critical, since the system's dynamics and configuration can dramatically change over the course of time, possibly requiring quite different time step sizes to control the iterative error. However, to maintain the accuracy of the time-stepping scheme, one must respect an upper bound dictated by the discretization error, i.e., $\Delta t \leq \Delta t^{\text {lim }}$. Note that in step (5), $\Lambda_{K}$ may enlarge the time-step if the error is lower than the preset tolerance. At a given time, once the process is complete, then the time is incremented forward and the process is repeated. The overall goal is to deliver solutions where the iterative error is controlled and the temporal discretization accuracy dictates the upper limit on the time step size $\left(\Delta t^{l i m}\right)$. Clearly, there are various combinations of solution methods that one can choose from. For example, for the overall field coupling, one may choose implicit or explicit staggering and within the staggering process, either implicit $(0<\phi \leq 1)$ or explicit time-stepping $(\phi=0)$, and, in the case of implicit timestepping, iterative or direct solvers for the Navier-Stokes equations. Furthermore, one could employ internal iterations for each field equation, then update, more sophisticated metrics for certain components of the error, etc, etc. For example, we utilized an error measure that used the velocities at the nodes of the Finite Difference grid, which were partially induced by the particles. We could have isolated the error in velocities of the particles, used a combined metric, etc, etc. For details see Zohdi [50,51,55,56,58-62] (Fig. 5).

Remark 1 To accelerate the multi-particle calculations, one can utilize nearest neighbor interaction lists, which indicate with which neighboring particles a specific particle interacts, for a given number of time steps. All other particle-to-particle interaction is truncated. The interaction lists are updated periodically during the computations (see Pöschel and Schwa- 

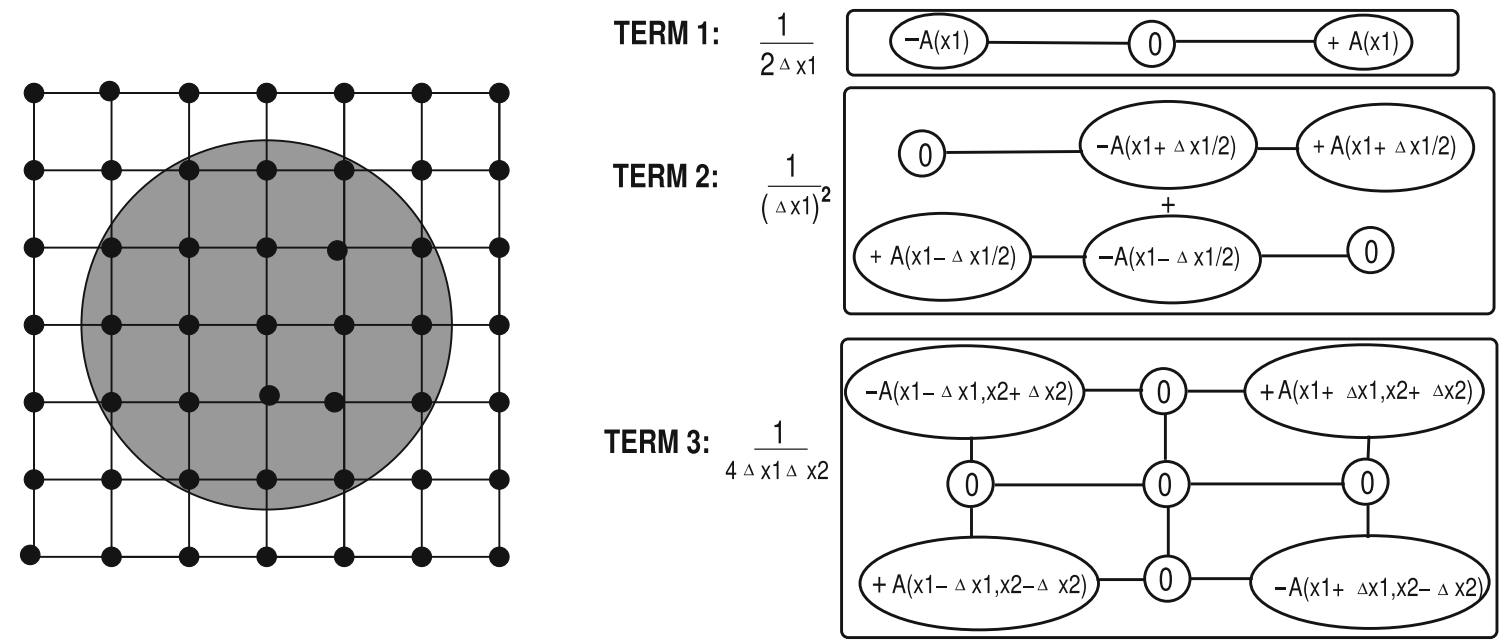

Fig. 5 Left Grid points where the average values of drag are computed. Right Various Finite-difference stencils in "computational molecule" form (centered at $\left.\left(x_{1}, x_{2}, x_{3}\right)\right)$, where: (1) $A(x) \frac{\partial v}{\partial x_{1}},(2) \frac{\partial}{\partial x_{1}}\left(A \frac{\partial v}{\partial x_{1}}\right)$ and (3) $\frac{\partial}{\partial x_{2}}\left(A \frac{\partial v}{\partial x_{1}}\right)$

Fig. 6 The model problem with the fluid boundary conditions fixed, while for the particles periodicity was imposed; i.e. any particle that exited from the domain was re-inserted at the opposite location from where they exited, but with the previous exit velocity
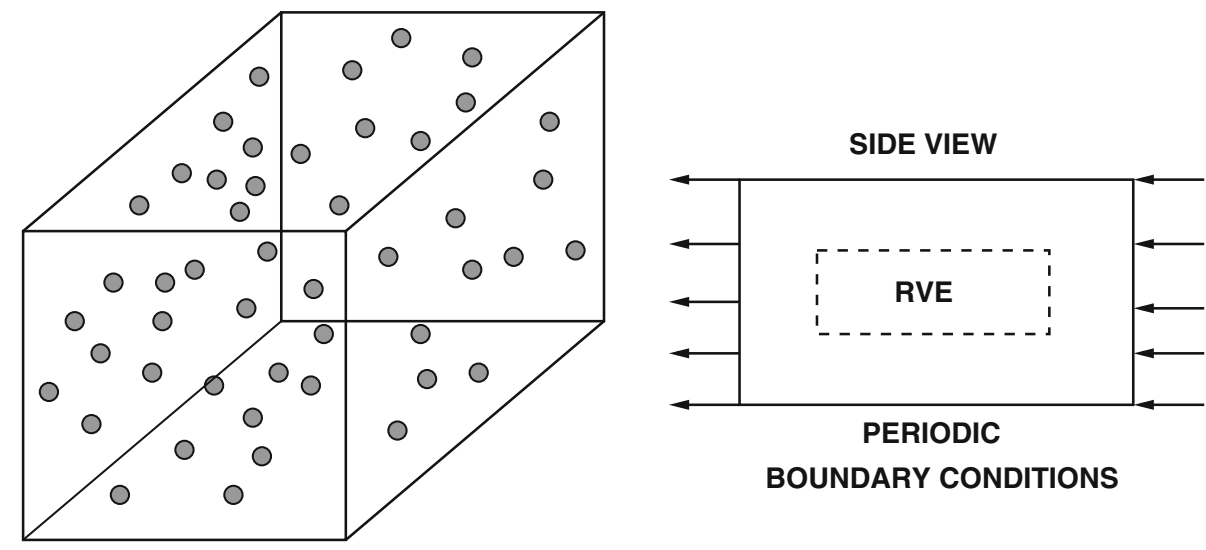

ger [29]). Such lists were used in the upcoming calculations and follow this procedure: (1) an initial full-blown contact search is performed at the beginning of the simulation and a list of nearest neighbors for each particle is generated, (2) the nearest neighbor lists are used for all inter-particle calculations for a preset subinterval of the total simulation time, $0 \leq \Delta T \leq T$ and (3) after a subinterval of the total simulation time, $0 \leq \Delta T \leq T$, elapses, the nearest neighbor list for each particle is updated. This type of link list strategy was used throughout the simulations. The results were repeatedly tested against simulations utilizing direct calculations (no list list truncation) to determine the proper settings for the link lists to ensure insensitivity to the truncations.

Remark 2 As the physics changes, the field that is most sensitive (exhibits the largest amount of relative nondimensional change during the iterations) dictates the time-step size. Because the internal system solvers within the staggering scheme are also iterative and use the previously converged solution as their starting value to solve the system of equa- tions, a field that is relatively insensitive at given stage of the simulation will converge in very few internal iterations (perhaps even one).

\section{Some examples}

We return to the system parameters that were used for a single particle, however, considering a group of $N_{p}$ particles. Specifically, we considered a group of $N_{p}=1000$ randomly positioned, non-overlapping, particles (Fig. 6). The rectangular domain was (length twice the width) using an $81 \times 41 \times 41$ finite difference grid (approximately 410000 nodal degrees of freedom), with an iterative tolerance set to $T O L=0.001$. The particle radii (monodisperse) were $\mathcal{R}=0.001 \mathrm{~m}$. The absolute dimensions are unimportant for the model problem, and have been normalized (a unit RVE domain). All system parameters can be scaled to describe any specific system of interest. The fluid boundary conditions were fixed for the fluid, while for the particles periodicity was imposed; i.e. any 
particle that exited from the domain was re-inserted at the opposite location from where they exited, but with it's previous exit velocity as its inlet velocity. The relevant simulation parameters, for illustration purposes only, were (implicitly using standard SI-units):

- The fluid viscosity, $\mu=0.13$,

- The particle density, $\rho_{p}=5000$,

- The drag coeffcient, $C_{D}=0.3$,

- The initial fluid velocity, $\boldsymbol{v}_{f}=(0.01,0,0)$,

- The fluid density, $\rho_{f}=2200$,

- The charge per unit mass, $\bar{q}=1$,

- The particle velocity, $\boldsymbol{v}_{p}(t=0)=(0.01,0,0)$,

- The coefficeint of static friction, $\mu_{s}=0.3$,

- The coefficient of dynamic friction, $\mu_{d}=0.2$,

- The normal contact compliance parameters: $K^{p}=0.01$, $p_{p}=2$,

- The friction contact compliance parameter: $K^{f}=0.01$,

- The target number of fixed point iterations, $K_{d}=10$ and

- The trapezoidal time-stepping parameter, $\phi=0.5$.

We considered various cases of electromagnetic field strengths. In order to calibrate the corresponding strength ratio of the electromagnetic forces to the drag forces, we evaluated the ratio introduced earlier $\left(\mathcal{L}\left(\boldsymbol{v}_{p}\right)\right)$, with a hypothetical value of $\boldsymbol{v}_{p}=0.5 \boldsymbol{v}_{f}(t=0)$ :

- Case $1 \boldsymbol{E}^{\text {ext }}=(0,0,0)$ and $\boldsymbol{B}^{\text {ext }}=(0,0,0) \Rightarrow \mathcal{L}\left(\boldsymbol{v}_{p}=\right.$ $\left.0.5 \boldsymbol{v}_{f}(t=0)\right)=0$

- Case $2 \boldsymbol{E}^{\text {ext }}=(0,0,1)$ and $\boldsymbol{B}^{\text {ext }}=(0,0,0) \Rightarrow \mathcal{L}\left(\boldsymbol{v}_{p}=\right.$ $\left.0.5 \boldsymbol{v}_{f}(t=0)\right)=8.08$

- Case $3 \boldsymbol{E}^{\text {ext }}=(0,0,1)$ and $\boldsymbol{B}^{\text {ext }}=(0,0,1) \Rightarrow \mathcal{L}\left(\boldsymbol{v}_{p}=\right.$ $\left.0.5 \boldsymbol{v}_{f}(t=0)\right)=8.12$

- Case $4 \boldsymbol{E}^{\text {ext }}=(0,0,1)$ and $\boldsymbol{B}^{\text {ext }}=(0,0,10) \Rightarrow \mathcal{L}\left(\boldsymbol{v}_{p}=\right.$ $\left.0.5 \boldsymbol{v}_{f}(t=0)\right)=8.48$

- Case $5 \boldsymbol{E}^{\text {ext }}=(0,0,1)$ and $\boldsymbol{B}^{\text {ext }}=(0,0,100) \Rightarrow$ $\mathcal{L}\left(\boldsymbol{v}_{p}=0.5 \boldsymbol{v}_{f}(t=0)\right)=12.12$ and

- Case $6 \boldsymbol{E}^{\text {ext }}=(0,0,1)$ and $\boldsymbol{B}^{\text {ext }}=(0,0,1000) \Rightarrow$ $\mathcal{L}\left(\boldsymbol{v}_{p}=0.5 \boldsymbol{v}_{f}(t=0)\right)=48.28$.

The cases illustrate that for increasing magnetic strength (Figs. 7, 8), a larger deviation in the $x_{1}$ velocity components of the fluid and the particles, because the particle motion is primarily in the $x_{2}-x_{3}$ plane. The simulations were run in 2-3 min on a Mac Powerbook. The approach allows researchers to rapidly compute such systems with laptop/desktop resources. The presented analysis and model can provide a useful guide to designing and interpreting experiments which, due to the coupled and embedded nature of these systems is complex. We discuss this further in the next section.

\section{Summary and discussion}

The primary objective of this communication was to investigate the relative contributions of external electromagnetic fields and fluid drag on electromagnetically sensitive particles embedded in a neutral fluid. Qualitative semi-analytical estimates were made for the relative contributions of these effects to the particle dynamics. Numerically, a staggered scheme was developed to solve the coupled system whereby a fully implicit Finite-Difference discretization of the NavierStokes equations was used for the fluid and a direct particledynamics discretization was performed for the particles. Because of the extreme computational difficulty and expense of a conforming spatial discretization needed for large numbers of embedded particles, simplifying assumptions were made allowing a reduced order model for the coupling, based on semi-analytical computation of drag-coefficients for the coupling of the particles and the fluid with coarse meshes. The strongly-coupled system was solved iteratively within each time-step using a recursive staggering scheme, which employed temporal adaptivity to control the error.

The developed approach is straightforward to implement and can be easily incorporated within any standard computational fluid mechanics code based on finite difference, finite element, finite volume or discrete/particle element discretization (see Labra and Onate [23], Onate et al. [26,27], Rojek et al. [31]). However, while the computational model can provide qualitative a-priori information for further computationally intensive large-scale simulations, extensions are invariably going to require complex spatial discretization, which entails resolving particle-fluid interaction in detail. This is quite important if one is interested in particle clustering and agglomeration (see Zohdi [53,55]). Accordingly, it is important to revisit one of the primary simplifying assumptions, in the context of extensions and improvements.

\subsection{Further resolution of particle geometry}

One of the primary assumptions was to assume that each particle is small enough that their rotation with respect to their mass centers was deemed insignificant. More detailed analyses of fluid-particle interaction can be achieved in a direct, brute-force, numerical schemes, treating the particles as part of the fluid continuum (as another fluid or solid phase), and thus meshing them in a detailed manner. In such an approach (for example see Avci and Wriggers [4])

- A fluid-only problem is solved, with (instantaneous) boundary conditions of $\boldsymbol{v}_{f}(\boldsymbol{x})=\boldsymbol{v}_{p i}(\boldsymbol{x})$ at each point on the fluid-particle boundaries, where the velocity of the points on the boundary are given by

$$
\boldsymbol{v}_{p i}(\boldsymbol{x})=\boldsymbol{v}_{p i}^{c m}+\omega_{i} \times \boldsymbol{R}_{c m \rightarrow \operatorname{surf} .}(\boldsymbol{x}),
$$



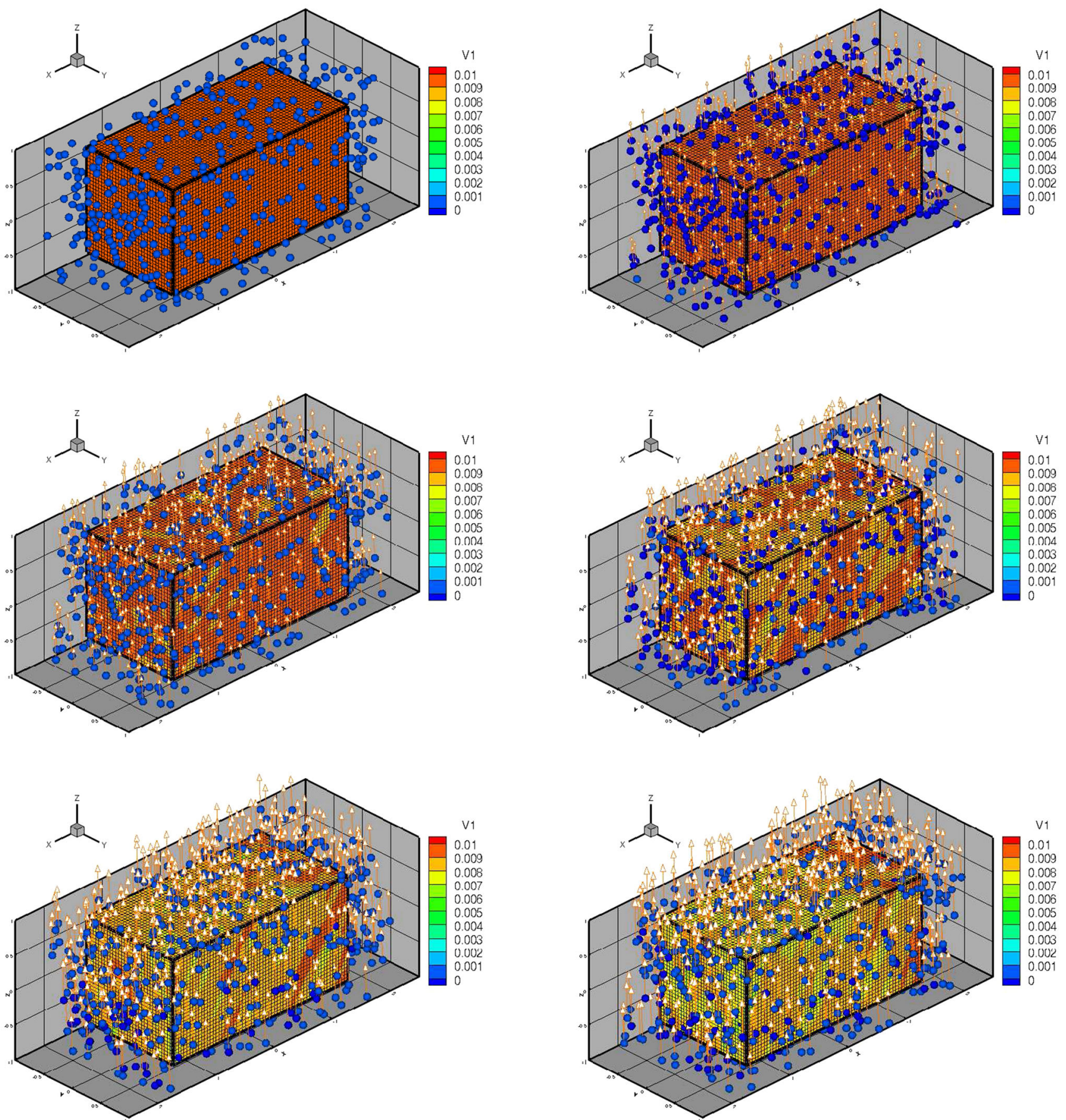

Fig. 7 Successive frames (left to right and top to bottom) for the progressive movement of particles. The $x_{1}$-component of the velocity for $\boldsymbol{E}^{\text {ext }}=(0,0,1)$ and $\boldsymbol{B}^{\text {ext }}=(0,0,1000)$

where $\boldsymbol{v}_{p i}^{c m}$ is the center of mass, for each of the individual particles and $\boldsymbol{R}_{c m \rightarrow \text { surf. }}$ is a vector from the mass center to the surface.

- For each particle, one would solve:

$$
m_{i} \dot{\boldsymbol{v}}_{p i}=\boldsymbol{\Psi}_{i}^{\text {drag }}+\text { other forces }
$$

and

$$
I_{i} \dot{\boldsymbol{\omega}}_{i}=\boldsymbol{M}_{i}^{\text {drag }}+\text { other moments }
$$

where the forces and moments would have a contribution from the fluid drag (with particle occupying domain $\Omega_{i}$ and outward surface normal $\boldsymbol{n}$ ) is defined 

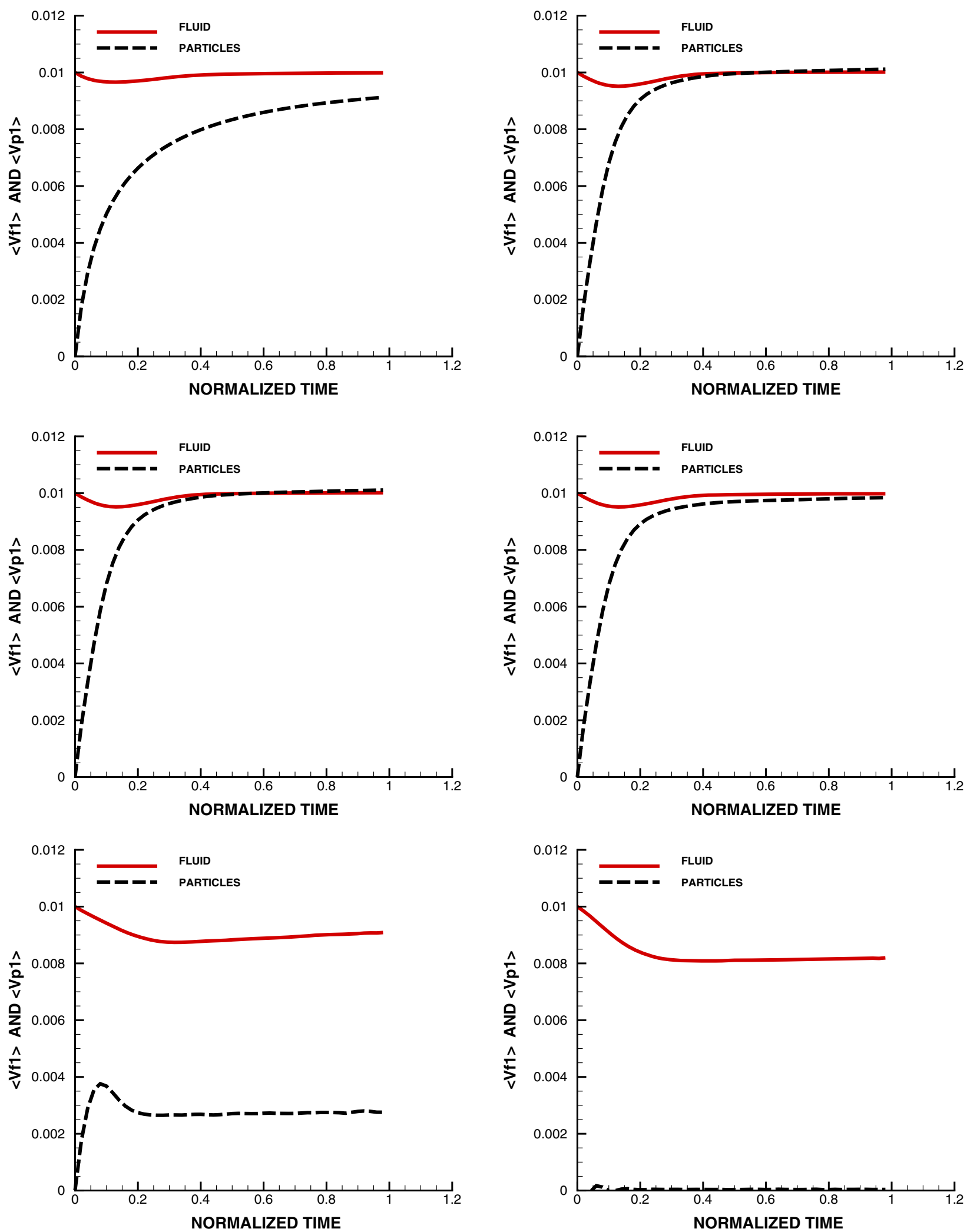

Fig. 8 The $x_{1}$-component of the velocity: Top Left: $\boldsymbol{E}^{\text {ext }}=(0,0,0)$ and $\boldsymbol{B}^{\text {ext }}=(0,0,0)$. Top Right: $\boldsymbol{E}^{\text {ext }}=(0,0,1)$ and $\boldsymbol{B}^{\text {ext }}=(0,0,0)$. Middle Left: $\boldsymbol{E}^{\text {ext }}=(0,0,1)$ and $\boldsymbol{B}^{\text {ext }}=(0,0,0)$. Middle Right:

$\boldsymbol{E}^{\text {ext }}=(0,0,1)$ and $\boldsymbol{B}^{\text {ext }}=(0,0,10)$. Bottom Left: $\boldsymbol{E}^{\text {ext }}=(0,0,1)$ and $\boldsymbol{B}^{\text {ext }}=(0,0,100)$. Bottom Right: $\boldsymbol{E}^{\text {ext }}=(0,0,1)$ and $\boldsymbol{B}^{\text {ext }}=$ $(0,0,1000)$ 
as

$$
\boldsymbol{\Psi}_{i}^{d r a g}=\int_{\partial \Omega_{i}} \boldsymbol{\sigma} \cdot \boldsymbol{n} d A
$$

and

$$
\boldsymbol{M}_{i}^{d r a g}=\int_{\partial \Omega_{i}} \boldsymbol{R}_{c m \rightarrow \operatorname{surf} .} \times \boldsymbol{\sigma} \cdot \boldsymbol{n} d A
$$

- At a time-step, the process is iteratively driven by solving the fluid-only problem first, then the particles-only problem, and repeated until convergence in an appropriate norm.

If one resorts to a direct brute force approach, then it is also probably appropriate to discard the assumption that the external electromagnetic fields are independently given. In other words, the action of the particle-laden fluid affects the electromagnetic fields. This requires a full resolution of Maxwell's equations, and we refer the reader to Zohdi $[59,60]^{9}$.

\subsection{Particle-wall interaction}

As mentioned in the outset, the long-term goal of this research is to develop experimentally-validated, predictive, computational models for the localized growth of interior wall coatings in damaged piping systems, controlled by electromagneto-mechanical means. The objective is to heal industrial piping systems noninvasively, from the exterior of the system, during operation, resulting in no down time with minimal relative cost. This type of analysis requires an electromagnetic model of the piping system walls, coupled to the flowing fluid (which contains charged particles). The relatively simple models that have been developed here provide a basis to start with experimental validation and refinement. A more complete model would entail coupling the fluid mechanics equations (Navier-Stokes) to the electromagnetic field (Maxwell) equations. For particle-wall interaction, this allows the associated electromagnetic force to be computed at the flowing-fluid solid interface. This force can impede or enhance the attachment of particle suspensions to the wall, since there are two primary effects that play a role at the charged solid-fluid interface/wall: (1) mechanical shear due to the fluid flow and (2) an electromagnetic force. The basic idea is that this electric force serves to manipulate the buildup of material in a piping system. Both electrical and magnetic approaches have been pursued in the realm of corro-

\footnotetext{
9 These fields are also of vastly different time-scales than those of the equations of particle-fluid motion.
}
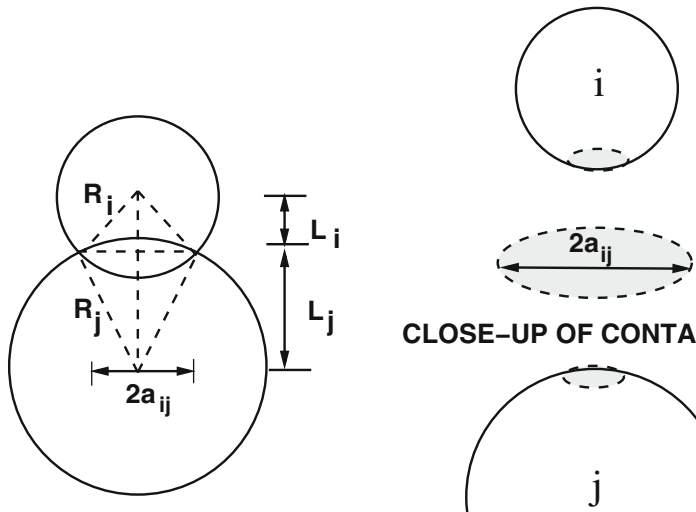

CLOSE-UP OF CONTACT AREA

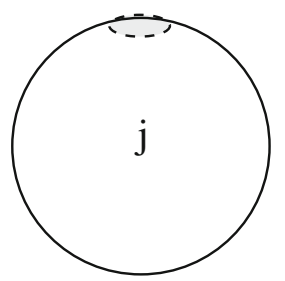

Fig. 9 An approximation of the contact area parameter for two particles in contact (after Zohdi [50])

sion and scale mitigation via electrochemical manipulation at the wall boundary. For example, see the extensive works of Waskaas and co-workers [28,36-39]. Regardless of the exact mechanism of effective growth, it is influenced by the intensity of the shear stress near the wall. High shear stresses tend to impede material from adhering to the wall, while low shear stresses allow for relatively more wall growth. As the coating grows, the flow past the selected growth location will be perturbed, and locally, there will be an increase in wall shear stress. The objective, as stated earlier, is to manipulate these effects to "heal" interior wall damage by producing thin layers which do not affect normal operation or produce costly system "down time". For this specific application, future work should concentrate on the development of a subsequent attachment and growth models, at walls involving both adhesion as well as wall growth. This is an area of some activity in biomechanics (see for example, for biological applications, Ambrosi et al. [2], Göktepe et al. [11], Hermanowicz [14-16], Sawyer and Hermanowicz [32], Menzel and Kuhl [24], Kuhl et al. [22], Yoon and Mofrad [49], Zöllner et al. [64], Zohdi et al. [52], Zohdi [53,54,57]). The adaptation of these types models to the problem of interest is currently being pursued by the author.

\section{Appendix 1: Contact area parameter}

Following Zohdi [50] and referring to Fig. 9, one can solve for an approximation of the common contact radius $a_{i j}$ (and the contact area, $A_{i j}^{c}=\pi a_{i j}^{2}$ ) by solving the following three equations:

$a_{i j}^{2}+L_{i}^{2}=\mathcal{R}_{i}^{2}$ 
and

$a_{i j}^{2}+L_{j}^{2}=\mathcal{R}_{j}^{2}$

and

$L_{i}+L_{j}=\left\|\boldsymbol{r}_{p i}-\boldsymbol{r}_{p j}\right\|$

where $\mathcal{R}_{i}$ is the radius of particle $i, \mathcal{R}_{j}$ is the radius of particle $j, L_{i}$ is the distance from the center of particle $i$ and the common contact interpenetration line and $L_{j}$ is the distance from the center of particle $j$ and the common contact interpenetration line, where the extent of interpenetration is

$\delta_{i j}=R_{i}+R_{j}-\left\|\boldsymbol{r}_{p i}-\boldsymbol{r}_{p j}\right\|$.

Solving these equations leads to

$A_{i j}^{c}=\pi a_{i j}^{2}=\pi\left(\mathcal{R}_{i}^{2}-L_{i}^{2}\right)$,

where

$L_{i}=\frac{1}{2}\left(\left\|\boldsymbol{r}_{p i}-\boldsymbol{r}_{p j}\right\|-\frac{\mathcal{R}_{j}^{2}-\mathcal{R}_{i}^{2}}{\left\|\boldsymbol{r}_{p i}-\boldsymbol{r}_{p j}\right\|}\right)$.

Remark One could can easily construct more elaborate relations connecting the relative proximity of the particles and other metrics to the contact force, $\boldsymbol{\Psi}_{i j}^{C} \propto$ $\mathcal{F}\left(\boldsymbol{r}_{i}, \boldsymbol{r}_{j}, \boldsymbol{n}_{i j}, R_{i}, R_{j}, \ldots\right)$, building on, for example, Hertzian contact models. This poses no difficulty in the direct numerical method developed. For much more detailed constitutive relations between particles. For the remainder of the analysis, we shall use the deformation metric in Eq. 5.3. We note that with the appropriate definition of parameters, one can recover Hertz, Bradley, Johnson-Kendel-Roberts, Derjaguin-MullerToporov contact models. For example, for normal Hertzian contact

$\Psi_{i j}^{\text {con }, n}=\frac{4}{3}\left(R^{*}\right)^{1 / 2} E^{*} \delta_{i j}^{3 / 2}$,

which has the general form of $\boldsymbol{\Psi}^{c o n, n}=K_{i j}^{*} \delta_{i j}^{p}$, where

- $R^{*}=\left(\frac{1}{R_{i}}+\frac{1}{R_{j}}\right)^{-1}$ and

- $E^{*}=\left(\frac{1-v_{i}^{2}}{E_{i}}+\frac{1-v_{j}^{2}}{E_{j}}\right)^{-1}$,

where $E$ is the Young's modulus and $v$ is the Poisson ratio, The contact area with such a model has already been incorporated in the relation above, and is equal to $A_{i j}^{C}=\pi a^{2}$ where $a=\sqrt{R^{*} \delta_{i j}}$. The assumptions in generating a Hertzian model are:

- frictionless, continuous, surfaces,

- each of contacting bodies are elastic half-spaces, whereby the contact area dimension are smaller radii of the bodies and,
- the bodies remain elastic (infinitesimal strains).

For more details, we refer the reader to Johnson [18]. For a more detailed understanding of the deformation of a particle a highly resolved discretization of the particle, for example using the Finite Element method would be needed (see Zohdi and Wriggers [58] and Wriggers [47,48]).

\section{Appendix 2: Inter-particle near-field interaction}

Following Zohdi [61], a simple form that captures the essential near-field features is

$$
\boldsymbol{\Psi}_{i}^{n f}=\underbrace{\sum_{j \neq i}^{n_{p}}(\underbrace{\boldsymbol{n}_{i j}}_{\text {attraction }},}_{\text {unit vector }}
$$

where the $\alpha$ 's and $\beta$ 's are empirical material parameters. The various possible representations (decompositions) of the coefficients that appear in Eq. 11.1 are with $c_{i}= \pm 1$ (a positive/negative identifier)

- $\operatorname{mass}-$ based ( $m=$ mass): $\alpha_{i j}=\bar{\alpha}_{i j} m_{i} m_{j} c_{i} c_{j}$,

- surface area-based ( $a=$ surface area): $\alpha_{i j}=\bar{\alpha}_{i j} a_{i} a_{j} c_{i} c_{j}$,

- volume-based ( $V=$ volume): $\alpha_{i j}=\bar{\alpha}_{i j} V_{i} V_{j} c_{i} c_{j}$ and

- charge-based: $\alpha_{i j}=\bar{\alpha}_{i j} q_{i} q_{j} c_{i} c_{j}$,

where the $\bar{\alpha}_{i j}$ are empirical material parameters. When a particle is relatively large, over essentially a millimeter, nearfields are quite small, in comparison with other forces. However, below one millimeter, a combination of the various near-field effects, positive and negative charge distributions, etc., can lead to a composite, or "effective" near-field, composed of an attractive and repulsive force (Eq. 11.1). These bear similarity to Molecular Dynamics (MD) type interaction laws. We refer the reader to Frenklach and Carmer [10]. In the usual MD approach (see Haile [12], for example), the motion of individual atoms is described by Newton's second law with the forces computed from differentiating a prescribed potential energy function, with applications to solids, liquids, and gases, as well as biological systems (Hase [13], Schlick [33] and Rapaport [30]). The interaction functions usually take the form of the familiar Mie, Lennard-Jones, and Morse potentials (Moelwyn-Hughes [25]), however threebody terms can be introduced directly into the interaction functions (Stillinger [34]) or, alternatively, "local" modifications can be made to two-body representations (Tersoff [35]) (Fig. 9). 


\section{Appendix 3: General governing fluid equations}

Recall, for a hydrostatic fluid the stress can be written as

$\sigma=-P_{o} \mathbf{1}$

where $P_{o}=\frac{t r \sigma}{3}$ is the hydrostatic pressure. In other words, there are no shear stresses in a fluid at rest. In the dynamic case, the pressure, denoted the "thermodynamic pressure", is related to the temperature and the fluid density by an equation of state

$\mathcal{Z}\left(P, \rho_{f}, \theta\right)=0$.

For a fluid in motion

$\sigma=-P \mathbf{1}+\boldsymbol{\tau}^{v s}$

where $\boldsymbol{\tau}^{v s}$ is a so-called viscous stress tensor, needed in a balance of linear momentum: ${ }^{10}$

$\nabla_{x} \cdot \boldsymbol{\sigma}+\boldsymbol{f}=\rho_{f} \frac{d \boldsymbol{v}_{f}}{d t}$,

where $\boldsymbol{v}_{f}$ is the fluid velocity at point $\boldsymbol{x}$ and $\boldsymbol{f}$ are the body forces. Thus, for a compressible fluid in motion:

$\frac{\operatorname{tr} \sigma}{3}=-P+\frac{\operatorname{tr} \tau^{v s}}{3}$.

In general, for a fluid we have

$\boldsymbol{\tau}^{v s}=\mathcal{G}(\boldsymbol{D})$ and $\boldsymbol{D} \stackrel{\text { def }}{=} \frac{1}{2}\left(\nabla_{x} \boldsymbol{v}_{f}+\left(\nabla_{x} \boldsymbol{v}_{f}\right)^{T}\right)$,

where $\boldsymbol{v}_{f}=\dot{\boldsymbol{u}}$ is the velocity and $\boldsymbol{D}$ is the symmetric part of the velocity gradient. For a Newtonian fluid, where a linear relation exists between the viscous stresses $\left(\boldsymbol{\tau}^{v s}\right)$ and $\boldsymbol{D}$

$\boldsymbol{\tau}^{v s}=\mathcal{G}(\boldsymbol{D})=\boldsymbol{C}: \boldsymbol{D}$

where $C$ is a symmetric positive definite (fourth-order) viscosity tensor. For an isotropic (standard) Newtonian fluid we have

$\sigma=-P \mathbf{1}+\lambda \operatorname{tr} \boldsymbol{D} \mathbf{1}+2 \mu \boldsymbol{D}=-P \mathbf{1}+3 \kappa \frac{\operatorname{tr} \boldsymbol{D}}{3} \mathbf{1}+2 \mu \boldsymbol{D}^{\prime}$,

where $\kappa$ is called the bulk viscosity, $\lambda$ is a viscosity constant, $\mu$ the shear viscosity and $\boldsymbol{D}^{\prime}=\boldsymbol{D}-\frac{\operatorname{tr} \boldsymbol{D}}{3} \mathbf{1}$. Explicitly, with an $\left(x_{1}, x_{2}, x_{3}\right)$ Cartesian triad

\footnotetext{
$\overline{10}$ An inviscid or "perfect" fluid is one where $\tau^{v s}$ is taken to be zero, even when motion is present.
}$$
\underbrace{\left\{\begin{array}{c}
\sigma_{11} \\
\sigma_{22} \\
\sigma_{33} \\
\sigma_{12} \\
\sigma_{23} \\
\sigma_{31}
\end{array}\right\}}_{\stackrel{\text { def }}{=}\{\boldsymbol{\sigma}\}}=\underbrace{\left\{\begin{array}{c}
-P \\
-P \\
-P \\
0 \\
0 \\
0
\end{array}\right\}}_{\stackrel{\text { def }}{=}\{-\boldsymbol{P}\}}+\underbrace{\left[\begin{array}{cccccc}
c_{1} & c_{2} & c_{2} & 0 & 0 & 0 \\
c_{2} & c_{1} & c_{2} & 0 & 0 & 0 \\
c_{2} & c_{2} & c_{1} & 0 & 0 & 0 \\
0 & 0 & 0 & \mu & 0 & 0 \\
0 & 0 & 0 & 0 & \mu & 0 \\
0 & 0 & 0 & 0 & 0 & \mu
\end{array}\right]}_{\stackrel{\text { def }}{=}[\boldsymbol{C}]} \underbrace{\left\{\begin{array}{c}
D_{11} \\
D_{22} \\
D_{33} \\
2 D_{12} \\
2 D_{23} \\
2 D_{31}
\end{array}\right\}}_{\stackrel{\text { def }}{=}\{\boldsymbol{D}\}}
$$

where $c_{1}=\kappa+\frac{4}{3} \mu$ and $c_{2}=\kappa-\frac{2}{3} \mu$, where $D_{i j}=$ $\frac{1}{2}\left(\frac{\partial v_{i}}{\partial x_{j}}+\frac{\partial v_{j}}{\partial x_{i}}\right)$. The so-called "Stokes' condition" attempts to force the thermodynamic pressure to collapse to the classical definition of mechanical pressure, i.e.

$\frac{\operatorname{tr} \sigma}{3}=-P+3 \kappa \frac{\operatorname{tr} \boldsymbol{D}}{3}=-P$,

leading to the conclusion that $\kappa=0$ or $\lambda=-\frac{2}{3} \mu$. Thus, a Newtonian fluid obeying the Stokes' condition has the following constitutive law:

$\boldsymbol{\sigma}=-P \mathbf{1}-\frac{2}{3} \mu t r \boldsymbol{D} \mathbf{1}+2 \mu \boldsymbol{D}=-P \mathbf{1}+2 \mu \boldsymbol{D}^{\prime}$.

Note that

$\dot{J}=\frac{d}{d t} \operatorname{det} \boldsymbol{F}=(\operatorname{det} \boldsymbol{F}) \operatorname{tr}\left(\dot{\boldsymbol{F}} \cdot \boldsymbol{F}^{-1}\right)=J \operatorname{tr} \boldsymbol{L}=J \nabla_{x} \cdot \boldsymbol{v}_{f}$,

where $\boldsymbol{L}=\nabla_{x} \boldsymbol{v}_{f}$ is the velocity gradient. Note that $\nabla_{x} \cdot \boldsymbol{v}_{f}=$ $\operatorname{tr} \boldsymbol{L}=\operatorname{tr} \boldsymbol{D}$. Therefore, if the fluid is incompressible, $\boldsymbol{j}=0$, then $\nabla_{x} \cdot \boldsymbol{v}_{f}=0=\operatorname{tr} \boldsymbol{L}=\operatorname{tr} \boldsymbol{D}$. Therefore,

$\boldsymbol{\sigma}=-P \mathbf{1}+2 \mu \boldsymbol{D}$.

A conservation of mass dictates

$\frac{d}{d t}\left(\rho_{f o}\right)=\frac{d}{d t}\left(\rho_{f} J\right)=J \frac{d \rho_{f}}{d t}+\rho_{f} \frac{d J}{d t}=0$,

which leads to

$\frac{d \rho_{f}}{d t}+\frac{\rho_{f}}{J} \frac{d J}{d t}=0$.

Using Eqs. 12.12, 12.14 becomes

$\frac{d \rho_{f}}{d t}+\rho_{f} \nabla_{x} \cdot \boldsymbol{v}_{f}=0$.

Now write the total temporal ("material") derivative in convective form:

$\frac{d \rho_{f}}{d t}=\frac{\partial \rho_{f}}{\partial t}+\left(\nabla_{x} \rho_{f}\right) \cdot \frac{d \boldsymbol{x}}{d t}=\frac{\partial \rho_{f}}{\partial t}+\nabla_{x} \rho_{f} \cdot \boldsymbol{v}_{f}$.

Thus, Eq. 12.12 becomes

$\frac{\partial \rho_{f}}{\partial t}+\nabla_{x} \rho_{f} \cdot \boldsymbol{v}_{f}+\rho_{f} \nabla_{x} \cdot \boldsymbol{v}_{f}=\frac{\partial \rho_{f}}{\partial t}+\nabla_{x} \cdot\left(\rho_{f} \boldsymbol{v}_{f}\right)=0$. 
Thus, writing the total time derivatives appearing previously as

$\frac{d \boldsymbol{v}_{f}}{d t}=\left.\frac{\partial \boldsymbol{v}_{f}}{\partial t}\right|_{\boldsymbol{x}}+\left.\left(\nabla_{x} \boldsymbol{v}_{f}\right)\right|_{t} \cdot \frac{d \boldsymbol{x}}{d t}$,

the coupled governing equations are (ignoring thermal effects)

$$
\begin{aligned}
& \frac{\partial \rho_{f}}{\partial t}=-\nabla_{x} \rho_{f} \cdot \boldsymbol{v}_{f}-\rho_{f} \nabla_{x} \cdot \boldsymbol{v}_{f} \\
& \rho_{f}\left(\frac{\partial \boldsymbol{v}_{f}}{\partial t}+\left(\nabla_{x} \boldsymbol{v}_{f}\right) \cdot \boldsymbol{v}_{f}\right)=\nabla_{x} \cdot \boldsymbol{\sigma}+\boldsymbol{f} \\
& \boldsymbol{\sigma}=-P \mathbf{1}+\lambda \operatorname{tr} \boldsymbol{D} \mathbf{1}+2 \mu \boldsymbol{D}=-P \mathbf{1}+3 \kappa \frac{\operatorname{tr} \boldsymbol{D}}{3} \mathbf{1}+2 \mu \boldsymbol{D}^{\prime}
\end{aligned}
$$

where, for example, $P$ is given by an Equation of State. Collectively, we refer to these equations as the "Navier-Stokes" equations. There are a total of three variables: $\rho_{f}, \boldsymbol{v}_{f}$, and $P$. It is customary to specify $\boldsymbol{v}_{f}$ and $P$ on the boundary, and to determine $\rho_{f}$ on the boundary through the Equation of State. It is important to emphasize that physically compatible boundary data must be applied, and this is not a trivial matter for compressible flow.

Remark 1 There are a variety of possible Equations of State that connect the density to the pressure, such as a Boussinesqlike relation, which is adequate to describe dense gases and fluids, derived from ${ }^{11}$

$\rho_{f} \approx \rho_{f o}\left(P_{o}\right)+\frac{\partial \rho_{f}}{\partial P} \Delta P$

where $\rho_{f_{o}}$ and $P_{o}$ are reference values and $\Delta P=P-P_{o}$. We define the bulk (compressibility) modulus by $\zeta \stackrel{\text { def }}{=} \rho_{f} \frac{\partial P}{\partial \rho_{f}}$, yielding

$\rho_{f} \approx \rho_{f o}\left(1+\frac{1}{\zeta} \Delta P\right) \Rightarrow P \approx P_{o}+\zeta\left(\frac{\rho_{f}}{\rho_{f o}}-1\right)$.

For a constant density case, $\rho_{f}=\rho_{f o}$, and utilizing the Boussinesq-like relation, $P=P_{o}$.

\section{References}

1. Albrecht T, Bhrer C, Fhnle M, Maier K, Platzek D, Reske J (1997) First observation of ferromagnetism and ferromagnetic domains in a liquid metal. Appl Phys A 65(2):215

2. Ambrosi D, Ateshian GA, Arruda EM, Cowin SC, Dumais J, Goriely A, Holzapfel GA, Humphrey JD, Kemkemer R, Kuhl E, Olberding JE, Taber LA, Garikipati K (2011) Perspectives on biological growth and remodeling. J Mech Phys Solids 59:863-883

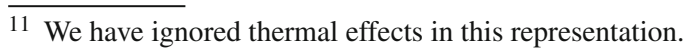

3. Andelman D, Rosensweig RE (2009) The phenomenology of modulated phases: from magnetic solids and fluids to organic films and polymers. In: Tsori Y, Steiner U Polymers, liquids and colloids in electric fields: interfacial instabilities, orientation and phase transitions. World Scientific, Singapore, p 156. ISBN 978-981-427168-4

4. Avci B, Wriggers P (2012) A DEM-FEM coupling approach for the direct numerical simulation of 3D particulate flows. J Appl Mech 79(1-7):010901

5. Berger P, Adelman NB, Beckman KJ, Campbell DJ, Ellis AB, Lisensky GC (1999) Preparation and properties of an aqueous ferrofluid. J Chem Educ 76(7):943-948. doi:10.1021/ed076p943 ISSN 0021-9584

6. Boyer TH (1988) The force on a magnetic dipole. Am J Phys 56(8):688-692. doi:10.1119/1.15501

7. Chow CY (1980) An introduction to computational fluid dynamics. Wiley, New York

8. Cullity BD, Graham CD (2008) Introduction to magnetic materials, 2nd edn. Wiley-IEEE Press, p 103. ISBN 0-471-47741-9

9. Feynman RP, Leighton RB, Sands M (2006) The Feynman Lectures on Physics 2. ISBN 0-8053-9045-6

10. Frenklach M, Carmer CS (1999) Molecular dynamics using combined quantum \& empirical forces: application to surface reactions. Adv Class Trajectory Methods 4:27-63

11. Göktepe S, Abilez OJ, Parker KK, Kuhl E (2010) A multiscale model for eccentric and concentric cardiac growth through sarcomerogenesis. J Theor Biol 265:433-442

12. Haile JM (1992) Molecular dynamics simulations: elementary methods. Wiley, Chichester

13. Hase WL (1999) Molecular dynamics of clusters, surfaces, liquids, \& interfaces. Advances in classical trajectory methods, vol. 4. JAI Press, Stamford

14. Hermanowicz SW (2004) Membrane filtration of biological solids : a unified framework and its applications to membrane bioreactors. In: Proceedings, water environment membrane technology 2004 conference, June 9, 2004, Seoul, South Korea

15. Hermanowicz SW (1999) Two-dimensional simulations of biofilm development: effects of external environmental conditions. Water Sci Technol 39(7):107-114

16. Hermanowicz SW (2001) A simple 2D biofilm model yields a variety of morphological features. Math Biosci 169(1):1-14

17. Jackson JD (1998) Classical electrodynamics, 3rd edn. Wiley, New York

18. Johnson K (1985) Contact mechanics. Cambridge University Press, Cambridge

19. Kaazempur-Mofrad MR, Younis HF, Patel S, Isasi AG, Chung C, Chan RC, Hinton DP, Lee RT, Kamm RD (2003) Cyclic strain in human carotid bifurcation and its potential correlation to atherogenesis: idealized and anatomically-realistic models. J Eng Math 47(3-4):299-314

20. Kaazempur-Mofrad MR, Younis HF, Isasi AG, Chan RC, Hinton DP, Sukhova G, LaMuraglia GM, Lee RT, Kamm RD (2004) Characterization of the atherosclerotic carotid bifurcation using MRI, finite element modeling and histology. Ann Biomed Eng 32(7):932-946

21. Kaazempur-Mofrad MR, Wada S, Myers JG, Ethier CR (2005) Blood flow and mass transfer in arteries with axisymmetric and asymmetric stenoses. Int J Heat Mass Transf 48:4510-4517

22. Kuhl E, Maas R, Himpel G, Menzel A (2007) Computational modeling of arterial wall growth: attempts towards patient specific simulations based on computer tomography. Biomech Model Mechanobiol 6:321-331

23. Labra C, Onate E (2009) High-density sphere packing for discrete element method simulations. Commun Numer Methods Eng 25(7):837-849 
24. Menzel A, Kuhl E (2012) Frontiers in growth and remodeling. Mech Res Commun 42:1-14

25. Moelwyn-Hughes EA (1961) Physical chemistry. Pergamon Press, Oxford

26. Onate E, Idelsohn SR, Celigueta MA, Rossi R (2008) Advances in the particle finite element method for the analysis of fluidmultibody interaction and bed erosion in free surface flows. Comput Methods Appl Mech Eng 197(19-20):1777-1800

27. Onate E, Celigueta MA, Idelsohn SR, Salazar F, Surez B (2011) Possibilities of the particle finite element method for fluid-soilstructure interaction problems. Comput Mech 48:307-318

28. Plausinaitis D, Waskaas M, Raudonis R, Daujotis V (2006) Piezoelectric resonator and high-frequency admittance study of viscosity and density changes in a thin water layer adjacent to the electrode surface. Electrochim Acta 51(27):6152-6158

29. Pöschel T, Schwager T (2004) Computational granular dynamics. Springer, Berlin

30. Rapaport DC (1995) The art of molecular dynamics simulation. Cambridge University Press, Cambridge

31. Rojek J, Labra C, Su O, Onate E (2012) Comparative study of different discrete element models and evaluation of equivalent micromechanical parameters. Int J Solids Struct 49:1497-1517. doi:10. 1016/j.ijsolstr.2012.02.032

32. Sawyer LK, Hermanowicz SW (2000) Detachment of Aeromonas hydrophila and Pseudomonas aeruginosa due to variations in nutrient supply. Water Sci Technol 41(4-5):139-145

33. Schlick T (2000) Molecular modeling and simulation. An interdisciplinary guide. Springer, New York

34. Stillinger FH, Weber TA (1985) Computer simulation of local order in condensed phases of silicon. Phys Rev B 31:5262-5271

35. Tersoff J (1988) Empirical interatomic potential for carbon, with applications to amorphous carbon. Phys Rev Lett 61:2879-2882

36. Waskaas $\mathrm{M}$ (2002) Method for reduction of flow resistance in pipes and ducts. United States Patent No. US 6,334,957 B1, 2002

37. Waskaas M (2006) Piezometric pressure measurements for water flow in a pipe with electrified inner surface. Russ J Electrochem 42(12):1340-1344(5)

38. Waskaas M, Matveyev H, Esbensen KH (2003) Novel discovery: flow improvement by a particular applied electrical DC-potential. An experimental study. Magnetohydrodynamics 39:501-505

39. Waskaas M, Daujotis V, Wolden KE, Raudonis R, Plausinaitis D (2008) Reduction in pressure drop for pipe flow due to applied electric potentials to the pipe. Elektrokhimiya 44(5):649-656

40. Wellmann C, Lillie C, Wriggers P (2008) A contact detection algorithm for superellipsoids based on the common-normal concept. Eng Comput 25:432-442

41. Wellmann C, Lillie C, Wriggers P (2008) Homogenization of granular material modelled by a three-dimensional discrete element method. Comput Geotech 35:394-405

42. Wellmann C, Lillie C, Wriggers P (2008) Comparison of the macroscopic behavior of granular materials modeled by different constitutive equations on the microscale. Finite Elem Anal Des 44:259 271

43. Wellmann C, Wriggers P (2012) A two-scale model of granular materials. Comput Methods Appl Mech Eng 205-208:46-58
44. Wellmann C, Wriggers P (2011) Homogenization of granular material modeled by a 3D DEM, particle-based methods: fundamentals and applications. In: Onate E, Owen DRJ (eds), p 211-231

45. Winslow WM (1947) Method and means for translating electrical impulses into mechanical force. U.S. Patent 2,417,850

46. Winslow WM (1949) Induced fibration of suspensions. J Appl Phys 20(12):1137-1140

47. Wriggers P (2002) Computational contact mechanics. Wiley, Chichester

48. Wriggers P (2008) Nonlinear finite element analysis. Springer, Berlin

49. Yoon SH, Chang J, Lin L, Mofrad MRK (2011) A biological breadboard platform for cell adhesion and detachment studies. Lab Chip 11(20):3555-3562

50. Zohdi TI (2014) A direct particle-based computational framework for electrically-enhanced thermo-mechanical sintering of powdered materials. Math Mech Solids 1-21. doi:10.1007/ s11831-013-9092-6

51. Zohdi TI (2002) An adaptive-recursive staggering strategy for simulating multifield coupled processes in microheterogeneous solids. Int J Numer Methods Eng 53:1511-1532

52. Zohdi TI, Holzapfel GA, Berger SA (2004) A phenomenological model for atherosclerotic plaque growth and rupture. J Theor Biol 227(3):437-443

53. Zohdi TI (2004) A computational framework for agglomeration in thermo-chemically reacting granular flows. Proc R Soc 460(2052):3421-3445

54. Zohdi TI (2005) A simple model for shear stress mediated lumen reduction in blood vessels. Biomech Model Mechanobiol 4(1):5761

55. Zohdi TI (2005) Charge-induced clustering in multifield particulate flow. Int J Numer Methods Eng 62(7):870-898

56. Zohdi TI (2007) Computation of strongly coupled multifield interaction in particle-fluid systems. Comput Methods Appl Mech Eng 196:3927-3950

57. Zohdi TI (2010) Charged wall-growth in channel-flow. Int J Eng Sci 48:15-20

58. Zohdi TI (2010) On the dynamics of charged electromagnetic particulate jets. Arch Comput Methods Eng 17(2):109-135

59. Zohdi TI (2010) Simulation of coupled microscale multiphysicalfields in particulate-doped dielectrics with staggered adaptive FDTD. Comput Methods Appl Mech Eng 199:79-101

60. Zohdi TI (2012) Electromagnetic properties of multiphase dielectrics. A primer on modeling, theory and computation. Springer, Dordrecht

61. Zohdi TI (2012) Dynamics of charged particulate systems. Modeling, theory and computation. Springer, Berlin

62. Zohdi TI (2013) Numerical simulation of charged particulate cluster-droplet impact on electrified surfaces. J Comput Phys 233:509-526

63. Zohdi TI (2014) Mechanically-driven accumulation of microscale material at coupled solid-fluid interfaces in biological channels. Proc R Soc Interface 11(91):20130922

64. Zöllner AM, Buganza Tepole A (2012) On the biomechanics and mechanobiology of growing skin. J Theor Bio 297:166-175 